\title{
CONVERGENCE ANALYSIS FOR FINITE ELEMENT DISCRETIZATIONS OF THE HELMHOLTZ EQUATION WITH DIRICHLET-TO-NEUMANN BOUNDARY CONDITIONS
}

\author{
J. M. MELENK AND S. SAUTER
}

\begin{abstract}
A rigorous convergence theory for Galerkin methods for a model Helmholtz problem in $\mathbb{R}^{d}, d \in\{1,2,3\}$ is presented. General conditions on the approximation properties of the approximation space are stated that ensure quasi-optimality of the method. As an application of the general theory, a full error analysis of the classical $h p$-version of the finite element method ( $h p$-FEM) is presented for the model problem where the dependence on the mesh width $h$, the approximation order $p$, and the wave number $k$ is given explicitly. In particular, it is shown that quasi-optimality is obtained under the conditions that $k h / p$ is sufficiently small and the polynomial degree $p$ is at least $O(\log k)$.
\end{abstract}

\section{INTRODUCTION}

Helmholtz boundary value problems appear in various applications, for example, in the context of inverse and scattering problems. When such problems are solved numerically, the questions of stability and convergence arise. Of particular interest is how critical parameters such as the discretization parameters (e.g., mesh size, approximation order) and the wave number $k$ affect the performance of the method.

Many discretization techniques for Helmholtz problems have been proposed and discussed in the literature. In the context of Galerkin methods, which is the setting of the present paper, these include both standard and non-standard finite element methods. Although significant progress in the understanding of the behavior of numerical methods for Helmholtz problems has been made in the past, a general, full analysis that is explicit in the wave number $k$ and discretization parameters is still not available. Partial results such as sharp estimates for the inf-sup constant of the continuous equations, lower estimates for the convergence rates, one-dimensional analysis by using the discrete Green's function as well as a dispersion analysis for finite element discretizations and generalizations thereof have been derived by many researchers in the past decades (see, e.g., 2, 4, 6, 7, 9, 10, 11, 15, 17, 18, 19, 22, 23, 24, 25, 26, 27, 28, 33, 38, 43, 44,).

The goal of the present and the companion paper [32] is to derive fairly general stability and convergence estimates for Helmholtz problems that are:

- explicit in the wave number, the mesh width, and the polynomial degree of the $h p$-FEM space;

Received by the editor July 15, 2008.

2010 Mathematics Subject Classification. Primary 35J05, 65N12, 65N30.

Key words and phrases. Helmholtz equation at high wave number, stability, convergence, $h p$ finite elements. 
- valid for problems in $d$ spatial dimensions, $d \in\{1,2,3\}$;

- only based on approximation properties of the (generalized) finite element space; the rationale behind this requirement is that it is easier to verify such an approximation property than to perform a full-fledged convergence analysis for a given approximation space.

These estimates require the development of new analytical tools and cannot be achieved in one stroke. As a first step, therefore, the present paper focuses on the Helmholtz equation in a bounded $d$-dimensional domain $\Omega$ with transparent boundary conditions, which we assume to be realized exactly with a Dirichlet-toNeumann map (DtN map) $T_{k}$. We will place special attention on the case where $\Omega$ is a ball since then the DtN map $T_{k}$ can be analyzed fairly explicitly. In this specific setting, we provide stability and convergence estimates of finite element discretizations that are explicit in the wave number, the mesh width, and the polynomial degree of the finite element space. The companion paper 32 will build upon the results of the present paper and will address more general situations such as the Helmholtz equation with Robin boundary conditions on smooth bounded domains or in convex polygons.

The outline of this paper is as follows: Section 2 formulates the model problem. Section 3 provides an analysis of the model problem. In particular, the $k$ dependence of the solution is made explicit (Lemmata 3.9, 3.5). Section 4 analyzes the discrete stability and states conditions on the properties of the approximation space to ensure quasi-optimality of the Galerkin scheme. For the case where $\Omega$ is a circle or a sphere, the conditions for stability and quasi-optimality are made fully explicit (Theorems 4.2, 4.3). Section 5 applies the results of Section 4 to the $h p$-version of the FEM. In particular, for the setting of Theorem 4.2 we show in Corollary [5.6 that quasi-optimality of the $h p$-FEM can be achieved under the assumption that

$$
\frac{k h}{p}+k\left(\frac{k h}{\sigma p}\right)^{p} \leq C
$$

where the constants $C, \sigma>0$ are sufficiently small but independent of $h, p$, and $k$. Several appendices conclude the paper: Appendix $\mathrm{A}$ provides detailed properties of Bessel functions that are needed in Section 3. Appendix B is concerned with $h p$-approximation of functions in the Sobolev spaces $H^{s}$; the novel feature of our results is its focus on simultaneous approximation in $L^{2}$ and $H^{1}$, which is an essential ingredient in our $k$-explicit bounds. Appendix $\mathrm{C}$ finally provides $h p$ approximation results for functions that are analytic. These latter approximation results are tailored to regularity properties of solutions of Helmholtz-type problems.

\section{Formulation of the model Helmholtz problem}

The Helmholtz problem in the full space $\mathbb{R}^{d}$ with Sommerfeld radiation condition is given by: Find $U \in H_{\text {loc }}^{1}\left(\mathbb{R}^{d}\right)$ such that

$$
\mid \begin{array}{ll}
\left(-\Delta-k^{2}\right) U=f & \text { in } \mathbb{R}^{d}, \\
\left|\frac{\partial U}{\partial r}-\mathrm{i} k U\right|=o\left(\|\mathbf{x}\|^{\frac{1-d}{2}}\right) & \|\mathbf{x}\| \rightarrow \infty
\end{array}
$$

is satisfied in a weak sense (cf. [35]). Here, $\partial / \partial r$ denotes the derivative in radial direction $\mathbf{x} /\|\mathbf{x}\|$. We assume throughout the paper that the wave number is positive 
and bounded away from zero, i.e.,

$$
k \geq k_{0}>0 .
$$

We assume that $f$ is local in the sense that there exists a bounded, simply connected domain $\Omega \subset \mathbb{R}^{d}$ that satisfies supp $f \subset \Omega$. The complement of $\Omega$ is denoted by $\Omega^{+}:=\mathbb{R}^{d} \backslash \bar{\Omega}$ and the interface by $\Gamma:=\bar{\Omega} \cap \overline{\Omega^{+}}$. Then (2.1) can be formulated in an equivalent way as a transmission problem by seeking functions $u \in H^{1}(\Omega)$ and $u^{+} \in H_{\text {loc }}^{1}\left(\Omega^{+}\right)$such that

$$
\begin{array}{cl}
\left(-\Delta-k^{2}\right) u=f & \text { in } \Omega, \\
\left(-\Delta-k^{2}\right) u^{+}=0 & \text { in } \Omega^{+}, \\
u=u^{+} \text {and } \partial u / \partial n=\partial u^{+} / \partial n & \text { on } \partial \Omega, \\
\left|\frac{\partial u^{+}}{\partial r}-\mathrm{i} k u^{+}\right|=o\left(\|\mathbf{x}\|^{\frac{1-d}{2}}\right) & \|\mathbf{x}\| \rightarrow \infty .
\end{array}
$$

Here, $n$ denotes the normal vector pointing into the exterior domain $\Omega^{+}$.

It can be shown that, for given $g \in H^{1 / 2}(\partial \Omega)$, the problem

$$
\text { Find } w \in H_{\mathrm{loc}}^{1}\left(\Omega^{+}\right) \text {such that }\left\{\begin{array}{cl}
\left(-\Delta-k^{2}\right) w=0 & \text { in } \Omega^{+}, \\
w=g & \text { on } \partial \Omega, \\
\left|\frac{\partial w}{\partial r}-\mathrm{i} k w\right|=o\left(\|\mathbf{x}\|^{\frac{1-d}{2}}\right) & \|\mathbf{x}\| \rightarrow \infty
\end{array}\right.
$$

has a unique weak solution. The mapping $g \mapsto w$ is called the Steklov-Poincaré operator and is denoted by $S_{P}: H^{1 / 2}(\partial \Omega) \rightarrow H_{\mathrm{loc}}^{1}\left(\Omega^{+}\right)$. The Dirichlet-to-Neumann map is given by $T_{k}:=\gamma_{1} S_{P}: H^{1 / 2}(\partial \Omega) \rightarrow H^{-1 / 2}(\partial \Omega)$, where $\gamma_{1}:=\partial / \partial n$ is the normal trace operator. Hence, problem (2.3) can be reformulated as: Find $u \in H^{1}(\Omega)$ such that

$$
\begin{array}{cl}
\left(-\Delta-k^{2}\right) u=f & \text { in } \Omega, \\
\partial u / \partial n=T_{k} u & \text { on } \partial \Omega .
\end{array}
$$

The weak formulation of this equation is given by: Find $u \in H^{1}(\Omega)$ such that

$$
a(u, v):=\int_{\Omega}\langle\nabla u, \nabla \bar{v}\rangle-k^{2} u \bar{v}-\int_{\partial \Omega}\left(T_{k} u\right) \bar{v}=\int_{\Omega} f \bar{v} \quad \forall v \in H^{1}(\Omega) .
$$

The exact solution of (2.1) can be written as the acoustic volume potential. Let $G_{k}: \mathbb{R}^{d} \backslash\{0\} \rightarrow \mathbb{C}$ denote the fundamental solution to the operator $\mathcal{L}_{k}:=-\Delta-k^{2}$, i.e., $G_{k}(z)=g_{k}(\|z\|)$, where

$$
g_{k}(r):= \begin{cases}-\frac{\mathrm{e}^{\mathrm{i} k r}}{2 \mathrm{i} k} & d=1, \\ \frac{\mathrm{i}}{4} H_{0}^{(1)}(k r) & d=2, \\ \frac{\mathrm{e}^{\mathrm{i} k r}}{4 \pi r} & d=3 .\end{cases}
$$

Then, the solution of (2.1) is given by

$$
U(x):=\left(N_{k} f\right)(x):=\int_{\Omega} G_{k}(x-y) f(y) d y \quad \forall x \in \mathbb{R}^{d} .
$$

Consequently, the solution of (2.5) and (2.6) is given by

$$
u(x):=\left(N_{k} f\right)(x):=\int_{\Omega} G_{k}(x-y) f(y) d y \quad \forall x \in \Omega .
$$


Finally, we recall that a Galerkin method for (2.6) is given as follows: For a (typically finite dimensional) space $S \subset H^{1}(\Omega)$, the Galerkin approximation $u_{S} \in S$ to the exact solution $u$ is given by:

Find $u_{S} \in S$ s.t. $\quad a\left(u_{S}, v\right)=\int_{\Omega} f \bar{v} \quad \forall v \in S$.

\section{Analysis of the CONTINUOUS PROBlem}

The analysis of the continuous problem is split into three parts. First, we provide some estimates for the Dirichlet-to-Neumann map $T_{k}$. Then, we prove some mapping properties of the solution operator and, finally, state the existence and uniqueness of the continuous problem.

3.1. Estimates for the $\mathbf{D t N}$ operator $T_{k}$. We equip the space $H^{1}(\Omega)$ with the norm

$$
\|u\|_{\mathcal{H}}:=\left(|u|_{H^{1}(\Omega)}^{2}+k^{2}\|u\|_{L^{2}(\Omega)}^{2}\right)^{1 / 2},
$$

which is obviously equivalent to the $H^{1}(\Omega)$-norm. For $d=1$, the boundary $\partial \Omega$ consists of the two endpoints of $\Omega$ and the $L^{2}(\partial \Omega)$ - and $H^{1 / 2}(\partial \Omega)$-scalar product and norm are understood as

$$
(u, v)_{L^{2}(\partial \Omega)}:=\sum_{x \in \partial \Omega} u(x) \overline{v(x)} \text { and }\|u\|_{L^{2}(\partial \Omega)}=\|u\|_{H^{1 / 2}(\partial \Omega)}=\sqrt{\sum_{x \in \partial \Omega}|u(x)|^{2}} .
$$

For Lipschitz domains, it is well known that a trace estimate holds.

Lemma 3.1. There exists a constant $C_{\mathrm{tr}}$ depending only on $\Omega$ and $k_{0}$ such that for all $u \in H^{1}(\Omega)$,

$$
\begin{aligned}
\|u\|_{H^{1 / 2}(\partial \Omega)} & \leq C_{\operatorname{tr}}\|u\|_{\mathcal{H}}, \\
\|u\|_{L^{2}(\partial \Omega)} & \leq C_{\operatorname{tr}}\|u\|_{L^{2}(\Omega)}^{1 / 2}\|u\|_{H^{1}(\Omega)}^{1 / 2}
\end{aligned}
$$

Corollary 3.2. For $u \in H^{1}(\Omega)$, we have

$$
\sqrt{k}\|u\|_{L^{2}(\partial \Omega)} \leq \tilde{C}_{\mathrm{tr}}\|u\|_{\mathcal{H}} \quad \text { with } \quad \tilde{C}_{\mathrm{tr}}:=\frac{C_{\mathrm{tr}}}{\sqrt{2}} \frac{\sqrt{1+k_{0}^{2}}}{k_{0}},
$$

where $k_{0}$ is as in (2.2).

Proof. There holds

$$
\begin{aligned}
k\|u\|_{L^{2}(\partial \Omega)}^{2} & \leq C_{\mathrm{tr}}^{2} k\|u\|_{L^{2}(\Omega)}\|u\|_{H^{1}(\Omega)} \leq \frac{C_{\mathrm{tr}}^{2}}{2}\left(k^{2}\|u\|_{L^{2}(\Omega)}^{2}+\|u\|_{H^{1}(\Omega)}^{2}\right) \\
& =\frac{C_{\mathrm{tr}}^{2}}{2}\left(\left(1+k^{2}\right)\|u\|_{L^{2}(\Omega)}^{2}+|u|_{H^{1}(\Omega)}^{2}\right) \leq \tilde{C}_{\mathrm{tr}}^{2}\|u\|_{\mathcal{H}}^{2} .
\end{aligned}
$$

Let $B_{r}(x)$ denote the open ball with radius $r$ about $x$. For $x=0$, we write $B_{r}$ short for $B_{r}(0)$. Since the right-hand side $f$ in (2.3) has compact support, we may always choose $\Omega$ as some ball $B_{R}$. In the following analysis we will always restrict our attention to this case and assume that

$$
R \geq R_{0}>0 .
$$

Lemma 3.3. Let (3.3) and (2.2) be satisfied. For $d=2$, we assume additionally that $k_{0} \geq 1$. Then, there exist constants $c, C>0$ that depend solely on $R_{0}$ and $k_{0}$ such that the following is true: 
(1)

(3.4 $) \quad\left|\left(T_{k} u, v\right)_{L^{2}\left(\partial B_{R}\right)}\right| \leq C\|u\|_{\mathcal{H}}\|v\|_{\mathcal{H}} \quad \forall u, v \in H^{1}\left(B_{R}\right)$.

(2) For $d \in\{2,3\}$ and all $u \in H^{1 / 2}\left(\partial B_{R}\right)$ the real and imaginary parts of $\left(T_{k} u, u\right)_{L^{2}\left(\partial B_{R}\right)}$ satisfy

$$
\begin{aligned}
&-\operatorname{Re}\left(T_{k} u, u\right)_{L^{2}\left(\partial B_{R}\right)} \geq c \frac{\|u\|_{L^{2}\left(\partial B_{R}\right)}^{2}}{R}, \\
& \operatorname{Im}\left(T_{k} u, u\right)_{L^{2}\left(\partial B_{R}\right)}>0 \quad \text { for } u \neq 0 .
\end{aligned}
$$

For $d=1$, instead of (3.4b), (3.4C), there holds

$$
\begin{aligned}
- & \operatorname{Re}\left(T_{k} u, u\right)_{L^{2}\left(\partial B_{R}\right)}=0, \\
& \operatorname{Im}\left(T_{k} u, u\right)_{L^{2}\left(\partial B_{R}\right)} \geq k\|u\|_{L^{2}\left(\partial B_{R}\right)}^{2} .
\end{aligned}
$$

Before proving Lemma 3.3 , we note the following corollary.

Corollary 3.4. There exists $C_{c}>0$ which depend only on $k_{0}$ and $R_{0}$ (cf. (2.2), (3.3)) such that for all $u, v \in H^{1}\left(B_{R}\right)$,

$$
|a(u, v)| \leq C_{c}\|u\|_{\mathcal{H}}\|v\|_{\mathcal{H}} .
$$

Proof. The estimate

$$
|a(u, v)| \leq|u|_{H^{1}\left(B_{R}\right)}|v|_{H^{1}\left(B_{R}\right)}+k^{2}\|u\|_{L^{2}\left(B_{R}\right)}\|v\|_{L^{2}\left(B_{R}\right)}+\left|\int_{\partial B_{R}}\left(T_{k} u\right) \bar{v}\right|
$$

is obvious. Hence, the assertion follows from Lemma 3.3

Proof of Lemma 3.3, Case $d=3$.

The Dirichlet data on $\partial B_{R}$ can be expanded according to

$$
u(x)=\sum_{\ell=0}^{\infty} \sum_{m=-\ell}^{\ell} u_{\ell}^{m} Y_{\ell}^{m}(\theta, \phi),
$$

where $(R, \theta, \phi)$ are the spherical coordinates for $x \in \partial B_{R}$ and the functions $Y_{\ell}^{m}$ are the standard spherical harmonics. The solution to the exterior homogeneous Helmholtz problem with Sommerfeld radiation conditions at infinity and prescribed Dirichlet data at $\partial B_{R}$ can be expanded in the form

$$
u(x)=\sum_{\ell=0}^{\infty} \sum_{m=-\ell}^{\ell} u_{\ell}^{m} Y_{\ell}^{m}(\theta, \phi) \frac{h_{\ell}^{(1)}(k r)}{h_{\ell}^{(1)}(k R)},
$$

where $(r, \theta, \phi)$ are the spherical coordinates of $x \in \mathbb{R}^{3} \backslash \overline{B_{R}}$. By taking the normal derivative at the boundary we end up with a representation of the Dirichlet-toNeumann map

$$
T_{k} u=\sum_{\ell=0}^{\infty} \sum_{m=-\ell}^{\ell} u_{\ell}^{m} Y_{\ell}^{m}(\theta, \phi) \frac{z_{\ell}(k R)}{R}
$$

with the functions $z_{\ell}(r):=r \frac{\left(h_{\ell}^{(1)}\right)^{\prime}(r)}{h_{\ell}^{(1)}(r)}$. These functions have been analyzed in 35. Theorem 2.6.1] where it is shown that

$$
1 \leq-\operatorname{Re}\left(z_{\ell}(r)\right) \leq \ell+1 \text { and } 0<\operatorname{Im}\left(z_{\ell}(r)\right) \leq r .
$$


(In [35, Theorem 2.6.1], only $\operatorname{Im} z_{\ell}(r) \geq 0$ is stated, while the strict positivity follows from the positivity of the function $q_{\ell}$ in [35, (2.6.34)].) It follows from (3.7) that

$$
\int_{\partial B_{R}}\left(T_{k} u\right) \bar{v}=\sum_{\ell=0}^{\infty} \sum_{m=-\ell}^{\ell} \frac{z_{\ell}(k R)}{R} u_{\ell}^{m} \frac{}{v_{\ell}^{m}}
$$

and from (3.8) we conclude that

$$
\begin{aligned}
\left|\operatorname{Re} \int_{\partial B_{R}}\left(T_{k} u\right) \bar{v}\right| & =\left|\sum_{\ell=0}^{\infty} \sum_{m=-\ell}^{\ell}\left\{\frac{\operatorname{Re} z_{\ell}(k R)}{R} \operatorname{Re}\left(u_{\ell}^{m} \overline{v_{\ell}^{m}}\right)-\frac{\operatorname{Im} z_{\ell}(k R)}{R} \operatorname{Im}\left(u_{\ell}^{m} \overline{v_{\ell}^{m}}\right)\right\}\right| \\
& \leq \frac{1}{R} \sum_{\ell=0}^{\infty} \sum_{m=-\ell}^{\ell}\left\{\left|\operatorname{Re} z_{\ell}(k R)\right|+\left|\operatorname{Im} z_{\ell}(k R)\right|\right\}\left|u_{\ell}^{m}\right|\left|v_{\ell}^{m}\right| \\
& \leq \frac{1}{R} \sum_{\ell=0}^{\infty} \sum_{m=-\ell}^{\ell}\{|\ell+1|+k R\}\left|u_{\ell}^{m}\right|\left|v_{\ell}^{m}\right| \\
& \leq C\left(R^{-1}\|u\|_{H^{1 / 2}\left(\partial B_{R}\right)}\|v\|_{H^{1 / 2}\left(\partial B_{R}\right)}+k\|u\|_{L^{2}\left(\partial B_{R}\right)}\|v\|_{L^{2}\left(\partial B_{R}\right)}\right) .
\end{aligned}
$$

Using Corollary 3.2 we get

$$
\left|\operatorname{Re} \int_{\partial B_{R}}\left(T_{k} u\right) \bar{v}\right| \leq C \tilde{C}_{\mathrm{tr}}^{2}\left(1+\frac{1}{R_{0} k_{0}}\right)\|u\|_{\mathcal{H}}\|v\|_{\mathcal{H}} .
$$

Repeating these steps for the imaginary part results in the same upper bound, and we get for some $C$ that depends only on $R_{0}$ and $k_{0}$ the estimate

$$
\left|\int_{\partial B_{R}}\left(T_{k} u\right) \bar{v}\right| \leq C\|u\|_{\mathcal{H}}\|v\|_{\mathcal{H}} .
$$

The lower estimate of the real part follows from

$-\operatorname{Re} \int_{\partial B_{R}}\left(T_{k} u\right) \bar{u}=\sum_{\ell=0}^{\infty} \sum_{m=-\ell}^{\ell} \frac{-\operatorname{Re} z_{\ell}(k R)}{R}\left|u_{\ell}^{m}\right|^{2} \geq \sum_{\ell=0}^{\infty} \sum_{m=-\ell}^{\ell} \frac{1}{R}\left|u_{\ell}^{m}\right|^{2}=\frac{\|u\|_{L^{2}\left(B_{R}\right)}^{2}}{R}$.

The upper estimate for the imaginary part is just a repetition of the previous arguments.

For the lower estimate of the imaginary part, we consider $u \in H^{1 / 2}\left(\partial B_{R}\right) \backslash\{0\}$. Hence, there exists $\left(m_{\star}, \ell_{\star}\right)$ in the expansion (3.5) so that $u_{\ell_{\star}}^{m_{\star}} \neq 0$. This leads to

$$
\operatorname{Im} \int_{\partial B_{R}}\left(T_{k} u\right) \bar{u}=\sum_{\ell=0}^{\infty} \sum_{m=-\ell}^{\ell} \frac{\operatorname{Im} z_{\ell}(k R)}{R}\left|u_{\ell}^{m}\right|^{2} \geq C\left|u_{\ell_{\star}}^{m_{\star}}\right|^{2}>0
$$

and the lower bound is proved.

Case $d=2$.

We expand the Dirichlet data on $\partial B_{R}$ in polar coordinates

$$
u(x)=\sum_{\ell \in \mathbb{Z}} u_{\ell} \mathrm{e}^{\mathrm{i} \ell \theta}
$$


where $(R, \theta)$ are the polar coordinates of $x \in \partial B_{R}$. It follows (see, e.g., [12, (2.10)]) that

$$
T_{k} u=\sum_{\ell \in \mathbb{Z}} u_{\ell} \frac{w_{\ell}(k R)}{R} \mathrm{e}^{\mathrm{i} \ell \theta} \quad \text { with } \quad w_{\ell}(r):=r \frac{\left(H_{|\ell|}^{(1)}\right)^{\prime}(r)}{H_{|\ell|}^{(1)}(r)} .
$$

Obviously, it is sufficient to analyze $w_{\ell}$ only for $\ell \in \mathbb{N}_{0}$. By decomposing $w_{\ell}$ into its real and imaginary part we get

$$
w_{\ell}=r \frac{J_{\ell}^{\prime} J_{\ell}+Y_{\ell}^{\prime} Y_{\ell}+\mathrm{i}\left(Y_{\ell}^{\prime} J_{\ell}-J_{\ell}^{\prime} Y_{\ell}\right)}{J_{\ell}^{2}+Y_{\ell}^{2}} .
$$

For the imaginary part, we obtain

$$
Y_{\ell}^{\prime} J_{\ell}-J_{\ell}^{\prime} Y_{\ell} \stackrel{[1]}{=} Y_{\ell-1} J_{\ell}-J_{\ell-1} Y_{\ell} \stackrel{19.27 .16]}{=} \frac{2}{\pi r} .
$$

We set $M_{\ell}:=\left|H_{\ell}^{(1)}\right|$ and obtain

$$
w_{\ell}=r \frac{J_{\ell}^{\prime} J_{\ell}+Y_{\ell}^{\prime} Y_{\ell}}{M_{\ell}^{2}}+\mathrm{i} \frac{2}{\pi M_{\ell}^{2}}=\frac{r}{2} \frac{\frac{d}{d r} M_{\ell}^{2}}{M_{\ell}^{2}}+\mathrm{i} \frac{2}{\pi M_{\ell}^{2}} .
$$

In the next step, we derive estimates for the coefficients $w_{\ell}$.

\section{Case $d=2$ and $\ell \in \mathbb{N}_{\geq 2}$.}

Let

$$
\begin{aligned}
M_{\ell, n}^{2}(r) & :=\frac{2}{\pi r} \sum_{m=0}^{n} \frac{\delta_{\ell, m}}{r^{2 m}} \quad \text { with } \quad \delta_{\ell, m}:=\frac{(2 m) ! \gamma_{\ell, m}}{(m !)^{2} 16^{m}} \text { and } \\
\gamma_{\ell, m} & :=\prod_{k=1}^{m}\left(4 \ell^{2}-(2 k-1)^{2}\right)
\end{aligned}
$$

and define $R_{\ell, n}^{M}:=M_{\ell}^{2}-M_{\ell, n}^{2}$. Note that

$$
\gamma_{\ell, \ell}=\frac{(4 \ell) !}{2^{2 \ell}(2 \ell) !} \geq 0 \quad \text { and } \quad \gamma_{\ell, \ell+1}=-(4 \ell+1) \gamma_{\ell, \ell}<0 .
$$

We conclude from [46, §13.75] that, for the choice $n=\ell-1 \geq 0$, there holds $R_{\ell, \ell-1}^{M}(r) \geq 0$. Thus,

$$
M_{\ell}^{2}(r) \geq M_{\ell, \ell-1}^{2}(r) \quad \forall r \geq 0 .
$$

Let $K_{\nu}$ be the modified Bessel function of order $\nu$. From [46, §13.75] we obtain

$$
N_{\ell}^{2}:=\frac{d}{d r} M_{\ell}^{2}=-\frac{16}{\pi^{2}} \int_{0}^{\infty} K_{1}(2 r \sinh t) \sinh t \cosh (2 \ell t) d t
$$

and

$$
\frac{\cosh (2 \ell t)}{\cosh t}=\sum_{m=0}^{n} \frac{\gamma_{\ell, m}}{(2 m) !} \sinh ^{2 m} t+\tilde{R}_{\ell, n}^{2} .
$$

If $n>\ell-3 / 2$, the remainder $\tilde{R}_{\ell, n}$ satisfies

$$
\tilde{R}_{\ell, n}^{2} \in \begin{cases}{\left[0, \frac{\gamma_{\ell, n+1}}{(2 n+2) !} \sinh ^{2(n+1)} t\right]} & \text { if } \gamma_{\ell, n+1}>0, \\ {\left[\frac{\gamma_{\ell, n+1}}{(2 n+2) !} \sinh ^{2(n+1)} t, 0\right]} & \text { otherwise. }\end{cases}
$$


We introduce

$$
\begin{aligned}
N_{\ell, n}^{2} & :=-\frac{16}{\pi^{2}} \sum_{m=0}^{n} \frac{\gamma_{\ell, m}}{(2 m) !} \int_{0}^{\infty} K_{1}(2 r \sinh t)(\cosh t)\left(\sinh ^{2 m+1} t\right) d t \\
& =-\frac{16}{\pi^{2}} \sum_{m=0}^{n} \frac{\gamma_{\ell, m}}{(2 m) !(2 r)^{2 m+2}} \int_{0}^{\infty} K_{1}(z) z^{2 m+1} d z \\
& =-\frac{2}{\pi r^{2}} \sum_{m=0}^{n}(2 m+1) \frac{\delta_{\ell, m}}{r^{2 m}}=\frac{d}{d r} M_{\ell, n}^{2} .
\end{aligned}
$$

Note that $M_{\ell}^{2}(r)$ is monotone decreasing for $r>0$ (cf. [37, §9-7.3]) and hence $N_{\ell}^{2}(r)<0$ for $r>0$. Thus,

$$
\left|N_{\ell}^{2}(r)\right|=-N_{\ell, n}^{2}(r)+R_{\ell, n}^{N} \quad \text { with } \quad R_{\ell, n}^{N}:=-N_{\ell}^{2}(r)+N_{\ell, n}^{2}(r)
$$

and $R_{\ell, n}^{N}$ has the explicit representation

$$
R_{\ell, n}^{N}(r)=\frac{16}{\pi^{2}} \int_{0}^{\infty} K_{1}(2 r \sinh t)(\sinh t)(\cosh t) \tilde{R}_{\ell, n}^{2}(t) d t .
$$

Note that sinh, cosh, and $K_{1}$ are positive on the positive real axes (cf. [1, 9.6.23]). We choose $n=\ell$ and obtain from (3.13) and (3.15) that $\tilde{R}_{\ell, \ell}(t)$ is negative for $t>0$ and hence

$$
\left|N_{\ell}^{2}(r)\right| \leq-N_{\ell, \ell}^{2}(r) \quad \forall r>0 .
$$

In summary, we have proved that

$$
\begin{aligned}
\left|\operatorname{Re} w_{\ell}\right| & \leq-\frac{r}{2} \frac{N_{\ell, \ell}^{2}}{M_{\ell, \ell-1}^{2}}=\frac{1}{2} \frac{\sum_{m=0}^{\ell}(2 m+1) \frac{\delta_{\ell, m}}{r^{2 m}}}{\sum_{m=0}^{\ell-1} \frac{\delta_{\ell, m}^{2 m}}{r^{2 m}}} \leq \frac{2 \ell-1}{2}+\frac{2 \ell+1}{2} \frac{\frac{\delta_{\ell, \ell}}{r^{2 \ell}}}{\frac{\delta_{, \ell-1}}{r^{2 \ell-2}}} \\
& =\frac{2 \ell-1}{2}+\frac{(4 \ell-1)\left(4 \ell^{2}-1\right)}{16 \ell r^{2}} .
\end{aligned}
$$

Hence, for $\ell \geq 2$ and $r \geq C_{1} \sqrt{\ell}$ we arrive at

$$
\left|\operatorname{Re} w_{\ell}\right| \leq \frac{2 \ell-1}{2}\left(1+\frac{9}{8 C_{1}^{2}}\right) .
$$

It remains to consider the case

$$
r \leq C_{1} \sqrt{\ell}
$$

We derive from (3.11) and [1,9.1.27]

$$
\left|\frac{r}{2} N_{\ell}^{2}(r)\right|=-\frac{r}{2} N_{\ell}^{2}(r)=\ell M_{\ell}^{2}(r)-r\left(J_{\ell-1} J_{\ell}+Y_{\ell} Y_{\ell-1}\right),
$$

and this leads to

$$
\left|\operatorname{Re} w_{\ell}\right|=\frac{\left|\frac{r}{2} N_{\ell}^{2}(r)\right|}{M_{\ell}^{2}(r)}=\ell-\frac{r\left(J_{\ell} J_{\ell-1}+Y_{\ell} Y_{\ell-1}\right)}{M_{\ell}^{2}(r)} .
$$

We deduce from [1, 9.5.2, 9.1.7, 9.1.9] that

$$
J_{\ell}(r)>0 \quad \text { and } \quad Y_{\ell}(r)<0 \quad \forall 0 \leq r \leq \ell
$$

and thus

$$
J_{\ell} J_{\ell-1}+Y_{\ell} Y_{\ell-1}>0 \quad \forall 0 \leq r \leq \ell-1 .
$$

If $C_{1} \leq 2^{-1 / 2}$, there holds $C_{1} \sqrt{\ell} \leq \ell-1$ for all $\ell \geq 2$, and we have proved $\left|\operatorname{Re} w_{\ell}\right| \leq \ell$. 
To derive a lower bound for $\left(-\operatorname{Re} w_{\ell}\right)$, we proceed as for (3.17) and obtain, for $r \geq k_{0}$,

$$
\begin{aligned}
(3.20)-\operatorname{Re} w_{\ell}(r) & \geq-\frac{r}{2} \frac{N_{\ell, \ell-1}^{2}(r)}{M_{\ell, \ell}^{2}(r)}=\frac{1}{2} \frac{\sum_{m=0}^{\ell-1}(2 m+1) \frac{\delta_{\ell, m}}{r^{2} m}}{\sum_{m=0}^{\ell} \frac{\delta_{\ell, m}}{r^{2 m}}} \geq \frac{1}{2} \frac{1}{1+\frac{\frac{\delta_{\ell, \ell}}{r^{2} \ell}}{(2 \ell-1) \frac{\ell_{\ell \ell-1}}{r^{2 \ell-2}}}} \\
& =\frac{1}{2} \frac{1}{1+\frac{4 \ell-1}{8 \ell r^{2}}} \geq \frac{1}{2} \frac{1}{1+\frac{1}{2 k_{0}^{2}}} .
\end{aligned}
$$

For the imaginary part of $w_{\ell}$ we get

$$
\operatorname{Im} w_{\ell}(r)=\frac{2}{\pi M_{\ell}^{2}(r)}>0 \quad \forall \ell \in \mathbb{N}_{0} \quad \forall r \geq k_{0}
$$

because $M_{\ell}^{2}$ is non-negative and decreasing for $r>0$ (cf. [37, §9-7.3]). For the upper bound, we combine [20, 8.479] with the fact that $M_{\ell}^{2}$ is decreasing to obtain for $\ell \in \mathbb{N}_{\geq 1}$,

$$
M_{\ell}^{2}(r) \geq \frac{2}{\pi r} \quad \forall r \geq 1
$$

Hence, the upper bound

$$
\operatorname{Im} w_{\ell}(r)=\frac{2}{\pi M_{\ell}^{2}(r)} \leq r
$$

follows.

Case $d=2$ and $\ell=0,1$.

For $\ell=0$, we use [46, $\S 13.75]$ and get

$$
M_{0}^{2}(r) \geq M_{0,1}^{2}(r)=\frac{2}{\pi r}\left(1-\frac{1}{8 r^{2}}\right) .
$$

For $d=2$, there holds $k_{0}>1 / 2$ by our assumptions and, thus, for $r \geq k_{0}$ we get

$$
M_{0}^{2}(r) \geq \frac{1}{\pi r} .
$$

The combination of (3.11) and (3.22) implies

$$
\left|\operatorname{Re} w_{\ell}(r)\right| \leq \frac{\pi r^{2}}{2}\left|N_{\ell}^{2}(r)\right| .
$$

We deduce from (3.16) (which is also valid for $\ell=0,1$ ) that

$$
\left|N_{\ell}^{2}(r)\right| \leq\left|N_{\ell, \ell}^{2}(r)\right| \leq \frac{2}{\pi r^{2}} \sum_{m=0}^{\ell}(2 m+1) \frac{\delta_{\ell, m}}{r^{2 m}} \leq \frac{2}{\pi r^{2}} \begin{cases}1 & \ell=0, \\ 1+\frac{9}{8 r^{2}} & \ell=1 .\end{cases}
$$

This implies, for $r \geq k_{0}$ (cf. (2.2)),

$$
\left|N_{\ell}^{2}(r)\right| \leq C \frac{2}{\pi r^{2}}
$$

where $C$ depends solely on $k_{0}$. Thus, for $\ell=0,1$,

$$
\left|\operatorname{Re} w_{\ell}\right| \leq C \leq C(\ell+1) .
$$

Since $M_{\ell}^{2}$ is monotone decreasing (see [37, $\left.\S 9-7.3\right]$ ), it follows from (3.10) that $\operatorname{Re} w_{\ell}(r)<0$ for all $r>0$. 
In (3.20) we have derived a lower bound for $\left(-\operatorname{Re} w_{\ell}\right)$ provided $\ell \geq 1$. It remains to consider the case $\ell=0$. The assumption on $k_{0}$ implies $r \geq k_{0} \geq \frac{1}{2} \sqrt{3}$ so that

$$
-\operatorname{Re} w_{0}(r) \geq-\frac{r}{2} \frac{N_{0,1}^{2}(r)}{M_{0,0}^{2}(r)}=\frac{1}{2}\left(1-\frac{3}{8 r^{2}}\right) \geq \frac{1}{4} .
$$

To summarize both cases, we have proved that

$$
0<c \leq-\operatorname{Re} w_{\ell}(r) \leq C(\ell+1) \quad \forall r \geq k_{0} \quad \forall \ell \in \mathbb{N}_{0},
$$

where $c, C$ only depends on $k_{0}$.

For the imaginary part, it remains (cf. (3.22) for $\left(\operatorname{Im} w_{0}\right)$ and employ (3.11) and (3.22b) to obtain

$$
\operatorname{Im} w_{0}=\frac{2}{\pi M_{0}^{2}} \leq 2 r .
$$

By proceeding as for $d=3$ (after (3.8) ) the estimates (3.4) follow from (3.24).

Case $d=1$.

For boundary values $\psi:\{-R, R\} \rightarrow \mathbb{R}$, the Dirichlet-to-Neumann operator is given by

$$
T_{k} \psi=\mathrm{i} k \psi \text {. }
$$

The trace theorem (in one dimension) leads to

$$
\begin{aligned}
\left|\operatorname{Re} \int_{\partial B_{R}}\left(T_{k} u\right) \bar{v}\right| & =\left|\operatorname{Re}\left(\mathrm{i} k \sum_{r= \pm R} u(r) \bar{v}(r)\right)\right| \\
& \leq k\left|\operatorname{Im} \sum_{r= \pm R} u(r) \bar{v}(r)\right| \leq k \sum_{r= \pm R}|u(r)||\bar{v}(r)| \\
& \stackrel{\text { Cor. } 3.2}{\leq} C\|u\|_{\mathcal{H}}\|v\|_{\mathcal{H}},
\end{aligned}
$$

where $C$ only depends on $R_{0}$ and $k_{0}$. By the same techniques we can estimate the

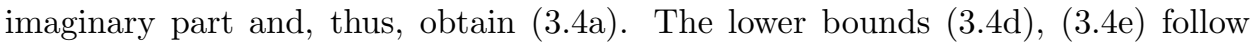
from

$$
\begin{gathered}
-\operatorname{Re} \int_{\partial B_{R}}\left(T_{k} u\right) \bar{u}=-\operatorname{Re}\left(\mathrm{i} k \sum_{r= \pm R}|u(r)|^{2}\right)=0, \\
\operatorname{Im} \int_{\partial B_{R}}\left(T_{k} u\right) \bar{u}=k \sum_{r= \pm R}|u(r)|^{2} \geq k\|u\|_{L^{2}\left(\partial B_{R}\right)}^{2} .
\end{gathered}
$$

3.2. Analysis of the solution operator $N_{k}$. In this section, we derive some explicit bounds for the solution operator $N_{k}$ under the assumption that the righthand side is in $L^{2}(\Omega)$. These estimates will be the basic tool for proving the discrete stability of the finite element discretization and the convergence. The key ingredient of the analysis of the $h p$-FEM in Section [5 is the following decomposition result:

Lemma 3.5 (decomposition lemma). Let $\Omega$ be contained in a ball of radius $R>0$. Then there exists a constant $C>0$ depending only on $R$ and $k_{0}$ such that for $f \in L^{2}(\Omega)$ the function $v$ given by

$$
v(x)=N_{k} f(x)=\int_{\Omega} G_{k}(x-y) f(y) d y, \quad x \in \Omega,
$$


satisfies

$$
k^{-1}\|v\|_{H^{2}(\Omega)}+\|v\|_{H^{1}(\Omega)}+k\|v\|_{L^{2}(\Omega)} \leq C\|f\|_{L^{2}(\Omega)} .
$$

Furthermore, for every $\lambda>1$, there exists a $\lambda$ - and $k$-dependent splitting $v=$ $v_{H^{2}}+v_{\mathcal{A}}$ with

$$
\begin{aligned}
& \text { (3.26) }\left\|\nabla^{p} v_{H^{2}}\right\|_{L^{2}(\Omega)} \leq C\left(1+\frac{1}{\lambda^{2}-1}\right)(\lambda k)^{p-2}\|f\|_{L^{2}(\Omega)} \quad \forall p \in\{0,1,2\}, \\
& \text { (3.26b) }\left\|\nabla^{p} v_{\mathcal{A}}\right\|_{L^{2}(\Omega)} \leq C \lambda(\sqrt{d} \lambda k)^{p-1}\|f\|_{L^{2}(\Omega)} \quad \forall p \in \mathbb{N}_{0} .
\end{aligned}
$$

Here, $\nabla^{p} v_{\mathcal{A}}$ stands for a sum over all derivatives of order $p$ (see (5.1) for details).

Remark 3.6. 1) For $f \in L^{2}(\Omega)$ the function $v=N_{k}(f)$ cannot be expected to have more Sobolev regularity than $H^{2}$. The decomposition $v=v_{H^{2}}+v_{\mathcal{A}}$ of Lemma 3.5 splits $v$ into an $H^{2}$-regular part $v_{H^{2}}$ and an analytic part $v_{\mathcal{A}}$. The essential feature of this splitting is that the $H^{2}$-part $v_{H^{2}}$ has a better $H^{2}$-regularity constant in terms of $k$ than $v$ itself, namely, (3.26回), (3.26b), and the triangle inequality $\left\|\nabla^{2} v\right\|_{L^{2}(\Omega)} \leq$ $\left\|\nabla^{2} v_{H^{2}}\right\|_{L^{2}(\Omega)}+\left\|\nabla^{2} v_{\mathcal{A}}\right\|_{L^{2}(\Omega)}$ imply

$$
\left\|\nabla^{2} v_{H^{2}}\right\|_{L^{2}(\Omega)} \leq C\|f\|_{L^{2}(\Omega)} \quad \text { versus } \quad\left\|\nabla^{2} v\right\|_{L^{2}(\Omega)} \leq C k\|f\|_{L^{2}(\Omega)} .
$$

The fact that $\left\|v_{H^{2}}\right\|_{H^{2}} \leq C\|f\|_{L^{2}}$ for a $C>0$ independent of $k$ will be essential for the stability and convergence analysis below.

2) Inspection of the proof shows that the mappings $f \mapsto v_{H^{2}}$ and $f \mapsto v_{\mathcal{A}}$ are linear maps.

Proof of Lemma 3.5. The estimates for $v$ follow directly from those for $v_{H^{2}}$ and $v_{\mathcal{A}}$ by fixing a parameter $\lambda>1$. In order to construct the splitting $v=v_{H^{2}}+v_{\mathcal{A}}$, we start by recalling the definition of the Fourier transform for functions with compact support

$$
\hat{u}(\xi)=(2 \pi)^{-d / 2} \int_{\mathbb{R}^{d}} \mathrm{e}^{-\mathrm{i}\langle\xi, x\rangle} u(x) d x \quad \forall \xi \in \mathbb{R}^{d}
$$

and the inversion formula

$$
u(x)=(2 \pi)^{-d / 2} \int_{\mathbb{R}^{d}} \mathrm{e}^{\mathrm{i}\langle x, \xi\rangle} \hat{u}(\xi) d \xi \quad \forall x \in \mathbb{R}^{d} .
$$

Let $B_{\Omega} \subset \mathbb{R}^{d}$ be a ball of radius $R$ containing $\Omega$. Extend $f$ by zero outside of $\Omega$ and denote this extended function again by $f$. Let $\mu \in C^{\infty}\left(\mathbb{R}_{\geq 0}\right)$ be a cutoff function such that

$$
\begin{array}{ll}
\operatorname{supp} \mu \subset[0,4 R], & \left.\mu\right|_{[0,2 R]}=1, \quad|\mu|_{W^{1, \infty}\left(\mathbb{R}_{\geq 0}\right)} \leq \frac{C}{R}, \\
\forall x \in \mathbb{R}_{\geq 0}: 0 \leq \mu(x) \leq 1,\left.\quad \mu\right|_{[4 R, \infty[}=0, \quad|\mu|_{W^{2, \infty}\left(\mathbb{R}_{\geq 0}\right)} \leq \frac{C}{R^{2}} .
\end{array}
$$

Define $M(z):=\mu(\|z\|)$ and

$$
v_{\mu}(x):=\int_{B_{\Omega}} G_{k}(x-y) M(x-y) f(y) d y \quad \forall x \in \mathbb{R}^{d} .
$$

The properties of $\mu$ guarantee $\left.v_{\mu}\right|_{B_{\Omega}}=\left.v\right|_{B_{\Omega}}$ so that we may restrict our attention to the function $v_{\mu}$. Since supp $f \subset B_{\Omega}$ we may write

$$
v_{\mu}=\left(G_{k} M\right) \star f,
$$


where " $\star$ " denotes the convolution in $\mathbb{R}^{d}$. We will define a decomposition of $v_{\mu}$ (which will determine the decomposition of $v$ on $B_{\Omega}$ ) by decomposing its Fourier transform, i.e.,

$$
\widehat{v}_{\mu}=\widehat{v}_{H^{2}}+\widehat{v}_{\mathcal{A}} .
$$

In order to define the two terms on the right-hand side of (3.29), we let $B_{\lambda k}(0)$ denote the ball of radius $\lambda k$ centered at the origin where $\lambda>1$ is the fixed constant (independent of $k$ ) selected in the statement of the lemma. The characteristic function of $B_{\lambda k}(0)$ is denoted by $\chi_{\lambda k}$. The Fourier transform of $f$ is then decomposed as

$$
\widehat{f}=\widehat{f} \chi_{\lambda k}+\left(1-\chi_{\lambda k}\right) \widehat{f}=: \widehat{f}_{k}+\widehat{f}_{k}^{c} .
$$

By the inverse Fourier transformation, this decomposition of $\widehat{f}$ entails a decomposition of $f$ into $f_{k}$ and $f_{k}^{c}$ given by

$$
f_{k}(x):=(2 \pi)^{-d / 2} \int_{\mathbb{R}^{d}} \mathrm{e}^{\mathrm{i}\langle x, \xi\rangle} \chi_{\lambda k}(\xi) \hat{f}(\xi) d \xi \quad \text { and } \quad f_{k}^{c}(x):=f-f_{k} .
$$

Accordingly, we define the decomposition of $v_{\mu}$ by

$$
v_{\mu, H^{2}}:=\left(G_{k} M\right) \star f_{k}^{c} \quad \text { and } \quad v_{\mu, \mathcal{A}}:=\left(G_{k} M\right) \star f_{k} .
$$

The functions $v_{H^{2}}$ and $v_{\mathcal{A}}$ in (3.29) are then obtained by setting $v_{H^{2}}:=\left.v_{\mu, H^{2}}\right|_{\Omega}$ and $v_{\mathcal{A}}:=\left.v_{\mu, \mathcal{A}}\right|_{\Omega}$. We will obtain the desired estimates by showing the following, stronger estimates:

$$
\begin{aligned}
\left\|v_{\mu, H^{2}}\right\|_{H^{2}\left(\mathbb{R}^{d}\right)} & \leq C\|f\|_{L^{2}\left(\mathbb{R}^{d}\right)}, \\
\left\|D^{\alpha} v_{\mu, \mathcal{A}}\right\|_{L^{2}\left(\mathbb{R}^{d}\right)} & \leq C \lambda(\lambda k)^{|\alpha|-1}\|f\|_{L^{2}\left(\mathbb{R}^{d}\right)}, \quad \forall \alpha \in \mathbb{N}_{0}^{d} .
\end{aligned}
$$

The estimates (3.32) are obtained by Fourier techniques. To that end, we compute the Fourier transform of $G_{k} M$ :

$$
\begin{aligned}
\left(\widehat{G_{k} M}\right)(\xi) & =(2 \pi)^{-d / 2} \int_{\mathbb{R}^{d}} \mathrm{e}^{-\mathrm{i}\langle\xi, x\rangle} G_{k}(x) M(x) d x \\
& =(2 \pi)^{-d / 2} \int_{0}^{\infty} g_{k}(r) \mu(r) r^{d-1}\left(\int_{\mathbb{S}_{d-1}} \mathrm{e}^{-\mathrm{i} r\langle\xi, \zeta\rangle} d S_{\zeta}\right) d r \\
& =(2 \pi)^{-d / 2} I(\xi) .
\end{aligned}
$$

The inner integral in $I(\xi)$ can be evaluated analytically 11 and $I(\xi)=\iota(\|\xi\|)$ with

$$
\iota(s)= \begin{cases}2 \int_{0}^{\infty} g_{k}(r) \mu(r) \cos (s r) d r & d=1, \\ 2 \pi \int_{0}^{\infty} g_{k}(r) \mu(r) r J_{0}(r s) d r & d=2, \\ 4 \pi \int_{0}^{\infty} g_{k}(r) \mu(r) r^{2} \frac{\sin (r s)}{(r s)} d r & d=3 .\end{cases}
$$

\footnotetext{
${ }^{1}$ This is trivial for $d=1$ and follows for $d=2$ from [20, (3.338)(4)]. For $d=3$, we use the formula

$$
\int_{\mathbb{S}^{2}} e^{-i\langle x, \hat{x}\rangle} Y_{\ell}^{m}(x) d x=g_{\ell}(\|\hat{x}\|) Y_{\ell}^{m}\left(\frac{\hat{x}}{\|\hat{x}\|}\right) \quad \text { with } \quad g_{\ell}(r)=(-i)^{\ell} 4 \pi j_{\ell}(r)
$$

(which follows by a comparison of [35, Section 3.2.4, formula (3.2.44) and (3.2.54)]) for $m=\ell=0$, where $Y_{0}^{0}=$ const and $g_{0}(r)=4 \pi \sin (r) / r$.
} 
Applying the Fourier transform to the convolutions (3.31) leads to

$$
\begin{aligned}
\widehat{v}_{\mu, H^{2}} & =(2 \pi)^{d / 2} \widehat{G_{k} M \hat{f}_{k}^{c}}=(2 \pi)^{d / 2} \widehat{G_{k} M} \widehat{f}\left(1-\chi_{\lambda k}\right), \\
\widehat{v}_{\mu, \mathcal{A}} & =(2 \pi)^{d / 2} \widehat{G_{k} M} \widehat{f}_{k}=(2 \pi)^{d / 2} \widehat{G_{k} M} \widehat{f} \chi_{\lambda k} .
\end{aligned}
$$

To estimate higher order derivatives of $v_{\mu, H^{2}}$ and $v_{\mu, \mathcal{A}}$ we define for a multi-index $\alpha \in \mathbb{N}_{0}^{d}$ the function $P_{\alpha}: \mathbb{R}^{d} \rightarrow \mathbb{R}^{d}$ by $P_{\alpha}(\xi):=\xi^{\alpha}$ and obtain, by using standard properties of the Fourier transformation and the support properties of $\chi_{\lambda k}$, for all $|\alpha| \leq 2$

$$
\begin{aligned}
\left\|\partial^{\alpha} v_{\mu, H^{2}}\right\|_{L^{2}\left(\mathbb{R}^{d}\right)} & =(2 \pi)^{d / 2}\left\|P_{\alpha} \widehat{G_{k} M}\left(1-\chi_{\lambda k}\right) \widehat{f}\right\|_{L^{2}\left(\mathbb{R}^{d}\right)} \\
& \leq(2 \pi)^{d / 2}\left(\max _{\xi \in \mathbb{R}^{d}:|\xi| \geq \lambda k}\left|P_{\alpha} I(\xi)\right|\right)\left\|\left(1-\chi_{\lambda k}\right) \widehat{f}\right\|_{L^{2}\left(\mathbb{R}^{d}\right)} \\
& \leq(2 \pi)^{d / 2}\left(\max _{s \geq \lambda k}\left|s^{|\alpha|} \iota(s)\right|\right)\|f\|_{L^{2}(\Omega)} .
\end{aligned}
$$

Lemma 3.7. (iv) implies for $|\alpha| \in\{0,1,2\}$

$$
\max _{s \geq \lambda k}\left|s^{|\alpha|} \iota(s)\right| \leq C(\lambda k)^{|\alpha|-2}\left(1+\frac{1}{\lambda^{2}-1}\right) .
$$

Thus,

$$
\left\|\partial^{\alpha} v_{H^{2}}\right\|_{L^{2}\left(B_{\Omega}\right)} \leq C(\lambda k)^{|\alpha|-2}\left(1+\frac{1}{\lambda^{2}-1}\right)\|f\|_{L^{2}(\Omega)}
$$

and (3.26a follows.

Completely analogously, we derive for all $\alpha \in \mathbb{N}_{0}^{d}$,

$$
\left\|\partial^{\alpha} v_{\mu, \mathcal{A}}\right\|_{L^{2}\left(\mathbb{R}^{d}\right)} \leq(2 \pi)^{d / 2}\left(\max _{0 \leq s \leq \lambda k}\left|s^{|\alpha|} \iota(s)\right|\right)\|f\|_{L^{2}(\Omega)} .
$$

We can complete the proof of the lemma using the bounds on the function $\iota$ given in Lemma 3.7, (v) below and using (5.1), (5.2).

Lemma 3.7. For the function $\iota$ defined in (3.34) the quantity $s^{m} \iota(s)$ can be estimated:

(i) for $m=0$ by

$$
|\iota(s)| \leq C \frac{R}{k}
$$

(ii) for $m=1$ by

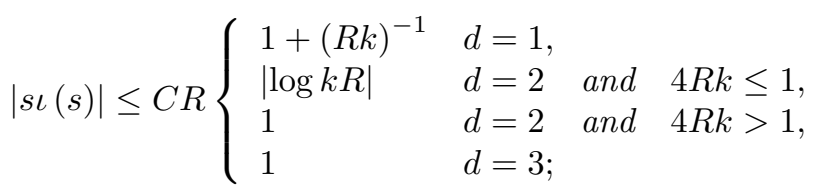

(iii) and for $m=2$ by

$$
s^{2}|\iota(s)| \leq C\left\{\begin{array}{lll}
R k+\frac{1}{R k} & d=1, & \\
|\log (k R)| & d=2 \\
R k & d=2 & \text { and } \quad 4 R k \leq 1, \\
1+k R & d=3 . &
\end{array}\right.
$$


(iv) For fixed $R_{0}, R_{1}>0$ there exists $C>0$ (depending only on $R_{0}, R_{1}, k_{0}, d$, and the constant appearing in (3.27)) such that for any $R \in\left[R_{0}, R_{1}\right]$ and any $\lambda>1$,

$$
\sup _{|s| \geq \lambda k} s^{2}|\iota(s)| \leq C\left(1+\frac{1}{\lambda^{2}-1}\right) .
$$

(v) For any $\lambda>0$ and all $m \in \mathbb{N}_{0}$ we have

$$
\sup _{|s| \leq \lambda k}|s|^{m}|\iota(s)| \leq C \lambda R(\lambda k)^{m-1} .
$$

Proof. In this proof, $C$ denotes a generic constant which may vary from term to term. It suffices to prove the estimates (3.7)-(3.7) because (3.7) follows directly from (3.7). We discuss the cases $d=3, d=1$, and $d=2$ in turn.

Case 1: $d=3$.

There holds

$$
|s \iota(s)|=C\left|\int_{0}^{\infty} \mathrm{e}^{\mathrm{i} k r} \mu(r) \sin (r s) d r\right| \leq C R .
$$

Applying integration by parts we obtain

$$
\begin{aligned}
|\iota(s)| & =\frac{C}{k}\left|\int_{0}^{\infty} \mathrm{e}^{\mathrm{i} k r}\left(\mu^{\prime}(r) \frac{\sin (r s)}{s}+\mu(r) \cos (r s)\right) d r\right| \\
& \leq \frac{C}{k} \int_{0}^{4 R}\left(\frac{C}{R} r+1\right) d r=C \frac{R}{k} .
\end{aligned}
$$

For the product $s^{2} \iota(s)$, we get

$$
\begin{aligned}
\left|s^{2} \iota(s)\right| & =C\left|\int_{0}^{\infty} \mathrm{e}^{\mathrm{i} k r} \mu(r) s \sin (r s) d r\right|=C\left|\int_{0}^{\infty} \mathrm{e}^{\mathrm{i} k r} \mu(r) \partial_{r} \cos (r s) d r\right| \\
& \leq C\left(\left|\int_{0}^{\infty} \cos (r s) \partial_{r}\left(\mathrm{e}^{\mathrm{i} k r} \mu(r)\right) d r\right|+1\right) \\
& \leq C k\left|\int_{0}^{\infty} \cos (r s) \mathrm{e}^{\mathrm{i} k r} \mu(r) d r\right|+C\left(\left|\int_{0}^{\infty} \cos (r s) \mathrm{e}^{\mathrm{i} k r} \mu^{\prime}(r) d r\right|+1\right) \\
& =: T^{\mathrm{I}}+T^{\mathrm{II}} .
\end{aligned}
$$

The estimates $T^{\mathrm{I}} \leq C^{\mathrm{I}} k R$ and $T^{\mathrm{II}} \leq C^{\mathrm{II}}$ follows from the properties of $\mu$ (cf. (3.27)). For $|s| \geq \lambda k$, the estimate of $T^{\mathrm{I}}$ can be refined by using integration by parts:

$$
\begin{aligned}
T^{\mathrm{I}} & \leq C k\left|\int_{0}^{\infty} \cos (r s) \mathrm{e}^{\mathrm{i} k r} \mu(r) d r\right|=C \frac{k}{2}\left|\int_{0}^{\infty}\left(\mathrm{e}^{\mathrm{i}(k+s) r}+\mathrm{e}^{\mathrm{i}(k-s) r}\right) \mu(r) d r\right| \\
& \leq C^{\prime}\left(\frac{k^{2}}{s^{2}-k^{2}}+\int_{0}^{\infty} \frac{k^{2}}{s^{2}-k^{2}}\left|\mu^{\prime}(r)\right| d r\right) \leq \frac{C^{\prime}(1+C)}{\lambda^{2}-1} .
\end{aligned}
$$

Case 2: $d=1$.

There holds

$$
|\iota(s)| \leq \frac{1}{k} \int_{0}^{\infty} \mu(r) d r \leq C \frac{R}{k}
$$


To estimate $s \iota(s)$, we apply integration by parts to obtain

$$
\begin{aligned}
|s \iota(s)| & \leq\left|\int_{0}^{\infty} \frac{\mathrm{e}^{\mathrm{i} k r}}{k} \mu(r) \partial_{r} \sin (s r) d r\right| \\
& =\left|\int_{0}^{\infty} \sin (s r) \partial_{r}\left(\frac{\mathrm{e}^{\mathrm{i} k r}}{k} \mu(r)\right) d r\right| \leq C \frac{1+R k}{k} .
\end{aligned}
$$

Similarly, we get by two-fold integration by parts:

$$
\begin{aligned}
\left|s^{2} \iota(s)\right| \leq & \left|\int_{0}^{\infty} \frac{\mathrm{e}^{\mathrm{i} k r}}{k} \mu(r) \partial_{r}^{2} \cos (s r) d r\right|=\left|\int_{0}^{\infty}\left\{\partial_{r} \cos (s r)\right\}\left\{\partial_{r}\left(\frac{\mathrm{e}^{\mathrm{i} k r}}{k} \mu(r)\right)\right\} d r\right| \\
\leq & \left|\int_{0}^{\infty} \cos (s r)\left\{\partial_{r}^{2}\left(\frac{\mathrm{e}^{\mathrm{i} k r}}{k} \mu(r)\right)\right\} d r+1\right| \\
\leq & k\left|\int_{0}^{\infty} \cos (s r) \mathrm{e}^{\mathrm{i} k r} \mu(r) d r\right| \\
& \quad+\left|\int_{0}^{\infty} \cos (s r)\left(2 \mathrm{i}^{\mathrm{i} k r} \mu^{\prime}(r)+\frac{\mathrm{e}^{\mathrm{i} k r}}{k} \mu^{\prime \prime}(r)\right) d r+1\right| \\
= & : T^{\mathrm{I}}+T^{\mathrm{III}} .
\end{aligned}
$$

The estimate $T^{\mathrm{III}} \leq C\left(1+\frac{1}{k R}\right)$ directly follows from the properties of the cutoff function $\mu$ (3.27). The term $T^{\mathrm{I}}$ was estimated already in Case 1 so that the proof of the case $d=1$ is complete.

Case 3a: $d=2$ and $4 R \leq 1 / k$.

For brevity, we write

$$
h_{k}(r):=H_{0}^{(1)}(k r) \quad \text { and } \quad j_{\nu, s}(r):=J_{\nu}(s r) .
$$

Estimate A.3C) implies

$$
\forall 0<r<4 R \leq 1 / k:\left|h_{k}(r)\right| \leq C(1+|\log k r|) \quad \text { and } \quad \forall r \geq 0:\left|J_{0}(r)\right|^{[1} \stackrel{9.1 .60]}{\leq} 1 .
$$

Hence,

$$
|\iota(s)| \leq C \int_{0}^{4 R}(1+|\log k r|) r d r=C R^{2}(1+|\log (4 k R)|) .
$$

For the estimate of $s^{m} \iota(s), m \in\{1,2\}$, we employ the relations (see [1, 9.1.30], [1, 9.1.1])

$$
\left(r j_{1, s}(r)\right)^{\prime}=r s j_{0, s}(r) \quad \text { and } \quad\left(r j_{0, s}^{\prime}(r)\right)^{\prime}=-r s^{2} j_{0, s}(r) .
$$

Integration by parts results in

$$
\begin{aligned}
|s \iota(s)| & \leq C\left|\int_{0}^{\infty} h_{k} \mu\left(r j_{1, s}\right)^{\prime} d r\right|=C\left|\int_{0}^{\infty} r j_{1, s}\left(\mu^{\prime} h_{k}+\mu h_{k}^{\prime}\right) d r\right| \\
& \leq \int_{0}^{4 k .8,} r\left\{\frac{(1+|\log k r|)}{R}+\frac{1}{r}+k^{2} r\right\} d r \\
& \leq C R\left\{1+|\log k R|+k^{2} R^{2}\right\} \leq C R(1+|\log k R|) \leq C R|\log k R| .
\end{aligned}
$$

Finally, we estimate $s^{2} \iota(s)$ by two-fold integration by parts (3.38)

$$
\left|s^{2} \iota(s)\right|=C\left|\int_{0}^{\infty} h_{k} \mu\left(r j_{0, s}^{\prime}\right)^{\prime}\right| \leq C\left(\left|\int_{0}^{4 R} j_{0, s}\left(r\left(h_{k} \mu\right)^{\prime}\right)^{\prime}\right|+\left|\lim _{r \rightarrow 0}\left(r h_{k}^{\prime}(r)\right)\right|\right) .
$$


Note that $\lim _{r \rightarrow 0} r h_{k}^{\prime}(r)=2 \mathrm{i} / \pi$. For the first term, we use

$$
\left(r\left(h_{k} \mu\right)^{\prime}\right)^{\prime}=\mu\left(r h_{k}^{\prime}\right)^{\prime}+2 r \mu^{\prime} h_{k}^{\prime}+\left(r \mu^{\prime}\right)^{\prime} h_{k} .
$$

We employ (A.12) for the first, (3.27), (A.11) for the second, (3.27), and (A.3) for the third term on the right-hand side in (3.39) to obtain

$$
\left|\left(r\left(h_{k} \mu\right)^{\prime}\right)^{\prime}\right| \leq C k^{2} r(1+|\log (k r)|)+\frac{1}{R}+\frac{R+r}{R^{2}}(1+|\log (k r)|) .
$$

Hence,

$$
\begin{aligned}
\left|s^{2} \iota(s)\right| & \leq C\left((k R)^{2}(1+|\log (k R)|)+1+|\log (k R)|\right) \\
& \leq C(1+|\log (k R)|) .
\end{aligned}
$$

Case 3b: $d=2$ and $4 R k>1$.

We define $\varphi_{k}(r):=h_{k}(r) \mu(r) r$ and denote its antiderivative by $\Phi_{k}(r):=$ $\int_{1 / k}^{r} \varphi_{k}(t) d t$. We use the splitting

$$
\iota(s)=\frac{\pi \mathrm{i}}{2} \int_{0}^{1 / k} \varphi_{k} j_{0, s}+\frac{\pi \mathrm{i}}{2} \int_{1 / k}^{4 R} \varphi_{k} j_{0, s}=: \iota_{\mathrm{I}}(s)+\iota_{\mathrm{II}}(s) .
$$

For $\iota_{\mathrm{I}}(s)$, we employ the estimates as in Case 3 a (with $4 R$ replaced by $1 / k$ therein) to obtain

$$
\left|\iota_{\mathrm{I}}(s)\right| \leq \frac{C}{k^{2}} .
$$

It remains to estimate $\iota_{\mathrm{II}}(s)$. Note that $j_{0, s}^{\prime}=-s j_{1, s}$. There holds

$$
\iota_{\text {II }}(s)=\frac{\pi \mathrm{i}}{2} \int_{1 / k}^{\infty} \varphi_{k} j_{0, s}=\frac{\pi \mathrm{i}}{2} \int_{1 / k}^{4 R} \Phi_{k} s j_{1, s}+\left.\frac{\pi \mathrm{i}}{2} \Phi_{k} j_{0, s}\right|_{r=1 / k} ^{4 R} .
$$

In the next step, we will estimate $\Phi_{k}$. Let $\tilde{\varphi}_{k}(r):=\mathrm{e}^{-\mathrm{i} k r} \varphi_{k}(r)$ so that $\Phi_{k}$ can be written as

$$
\begin{aligned}
\Phi_{k}(r) & :=\int_{1 / k}^{r} \mathrm{e}^{\mathrm{i} k t} \tilde{\varphi}_{k}(t) d t=-\int_{1 / k}^{r} \frac{\mathrm{e}^{\mathrm{i} k t}}{\mathrm{i} k} \tilde{\varphi}_{k}^{\prime}(t) d t+\left.\frac{\varphi_{k}(t)}{\mathrm{i} k}\right|_{t=1 / k} ^{r} \\
& =-\underbrace{\int_{1 / k}^{r} \frac{\mathrm{e}^{\mathrm{i} k t}}{\mathrm{i} k} \tilde{\varphi}_{k}^{\prime}(t) d t}_{=: \Phi_{k}^{\mathrm{I}}(r)}+\underbrace{\left.\frac{1}{\mathrm{i} k} t h_{k} \mu\right|_{t=1 / k} ^{r}}_{=: \Phi_{k}^{\mathrm{II}}(r)} .
\end{aligned}
$$

By using (A.6) and $\sup _{t>0}\left|(t \mu(t))^{\prime}\right| \leq C$ we obtain

$$
\begin{aligned}
\left|\Phi_{k}^{\mathrm{I}}(r)\right| & \leq \frac{1}{k} \int_{1 / k}^{r}\left|\tilde{\varphi}_{k}^{\prime}\right| d t=\frac{1}{k} \int_{1 / k}^{r}\left|t \mu\left(\mathrm{e}^{-\mathrm{i} k t} h_{k}\right)^{\prime}+(t \mu)^{\prime} \mathrm{e}^{-\mathrm{i} k t} h_{k}\right| d t \\
& \leq \frac{C}{k} \int_{1 / k}^{r} \frac{1}{\sqrt{k t}} d t \leq \frac{C}{k} \sqrt{\frac{r}{k}} .
\end{aligned}
$$

The function $\Phi_{k}^{\mathrm{II}}$ can be estimated by using (A.3․

$$
\left|\Phi_{k}^{\mathrm{II}}(r)\right|=\left|\frac{1}{\mathrm{i} k} t h_{k} \mu\right|_{t=1 / k}^{r} \mid \leq C\left(\frac{1}{k} \sqrt{\frac{r}{k}}+\frac{1}{k^{2}}\right)^{\sqrt{\frac{1}{k}} \leq \sqrt{r}} \frac{C}{k} \sqrt{\frac{r}{k}} .
$$

In summary we have proved

$$
\left|\Phi_{k}(r)\right| \leq \frac{C}{k} \sqrt{\frac{r}{k}}
$$


By inserting this estimate and (A.3b into (3.40) we get

$$
\begin{aligned}
\left|\iota_{\mathrm{II}}(s)\right| & \leq C \frac{\sqrt{|s|}}{k^{3 / 2}} \int_{0}^{4 R} \sqrt{\frac{|s| r}{1+r|s|}} d r+\left.\frac{C}{k} \sqrt{\frac{r}{k}}\right|_{r=4 R}+\left.\frac{C}{k} \sqrt{\frac{r}{k}}\right|_{r=1 / k} \\
& \leq C\left(\frac{4 R \sqrt{|s|}}{k^{3 / 2}}+\frac{1}{k} \sqrt{\frac{4 R}{k}}+\frac{1}{k^{2}}\right) .
\end{aligned}
$$

This leads to

$$
|\iota(s)| \leq C \frac{R}{k} \sqrt{\frac{|s|}{k}}+\frac{R}{k} .
$$

Next, we estimate $s^{2} \iota(s)$. As in the Case 3a, our starting point is (3.38). Recalling $\left|\lim _{r \rightarrow 0} r h_{k}^{\prime}(r)\right|=2 / \pi$, we are left with estimating

$$
\left|\int_{0}^{4 R} j_{0, s}\left(r\left(h_{k} \mu\right)^{\prime}\right)^{\prime}\right| \leq \underbrace{\left|\int_{0}^{1 / k} j_{0, s}\left(r\left(h_{k} \mu\right)^{\prime}\right)^{\prime}\right|}_{=: I_{1}}+\underbrace{\left|\int_{1 / k}^{4 R} j_{0, s}\left(r\left(h_{k} \mu\right)^{\prime}\right)^{\prime}\right|}_{=: I_{2}} .
$$

We conclude from Case 3a that $\left|I_{1}\right| \leq C$ holds. For the second integral, we employ (A.36), (3.27), (A.5), to get

$$
\begin{aligned}
\left|\left(r\left(h_{k} \mu\right)^{\prime}\right)^{\prime}\right| & =\left|h_{k}\left(\mu^{\prime}+r \mu^{\prime \prime}\right)+h_{k}^{\prime}\left(\mu+2 r \mu^{\prime}\right)+r h_{k}^{\prime \prime} \mu\right| \\
& \leq C\left(\frac{1}{r \sqrt{k r}}+\sqrt{\frac{k}{r}}+r k \sqrt{\frac{k}{r}}\right) \leq C\left(\frac{1}{r \sqrt{k r}}+k \sqrt{k r}\right) .
\end{aligned}
$$

The combination of (3.42), (3.43), and (A.3b) leads to

$$
I_{2} \leq C k R \sqrt{\frac{R k}{1+R|s|}}
$$

Thus, we have proved

$$
\left|s^{2} \iota(s)\right| \leq C k R \sqrt{\frac{R k}{1+R|s|}} .
$$

For $0 \leq|s| \leq k$, we employ (3.41) and for $|s|>k$ we use (3.44) to obtain for $m \in\{0,1,2\}$,

$$
|s|^{m}|\iota(s)| \leq C R k^{m-1} .
$$

For the case $d=2$, we now show (iv), i.e., we consider the case $|s| \geq \lambda k$. The assumptions $R \geq R_{0}$ and $k \geq k_{0}$ imply for the case $R k \leq 1 / 4$ immediately $\sup _{|s|>0} s^{2}|\iota(s)| \leq C$. For $R k>1 / 4$, we take, as in the Case $3 \mathrm{~b}$, the estimate (3.42) as our starting point. The integral $I_{1}$ in (3.42) is already seen to be bounded independent of $k$. Since, by [1, 9.1.1],

$$
\left(r h_{k}^{\prime}\right)^{\prime}=-k^{2} r h_{k}
$$

we can write the integral $I_{2}$ as

$$
I_{2}=\left|\int_{1 / k}^{4 R} j_{0, s}\left(-k^{2} r h_{k} \mu+2 r h_{k}^{\prime} \mu^{\prime}+\left(r \mu^{\prime}\right)^{\prime} h_{k}\right)\right| .
$$


Recalling that $\mu^{\prime} \equiv 0$ on $(0,2 R)$, we can estimate $I_{2}$ by

$$
I_{2} \leq \underbrace{\left|\int_{1 / k}^{4 R} j_{0, s} k^{2} r h_{k} \mu\right|}_{=: I_{2}^{I}}+C R \sup _{r \in(2 R, 4 R)}\left\{\left|j_{0, s} h_{k}^{\prime}\right|+\left|j_{0, s} h_{k}\right|\right\} .
$$

We conclude from (A.3), A.5 , and A.1) together with A.2

$$
C R \sup _{r \in(2 R, 4 R)}\left\{\left|j_{0, s} h_{k}^{\prime}\right|+\left|j_{0, s} h_{k}\right|\right\} \leq C R \frac{1}{\sqrt{|s| R}}\left(\frac{1}{R \sqrt{R k}}+\sqrt{\frac{k}{R}}+\frac{1}{\sqrt{R k}}\right) \leq C,
$$

where we used $|s| \geq \lambda k \geq k$ and the fact that $k \geq k_{0}$. It remains to bound $I_{2}^{I}$. Lemma A.1 allows us to write

$$
I_{2}^{I}=\frac{2 k^{2}}{\pi \sqrt{k|s|}}\left|\int_{1 / k}^{4 R} g^{I}(k r) \mu(r)\left\{e^{\mathrm{i}(|s|+k) r} f^{I}(|s| r)+e^{\mathrm{i}(-|s|+k) r} f^{I I}(|s| r)\right\}\right| .
$$

Since $f^{I}, f^{I I}, g^{I}$ are bounded functions by Lemma A.1 an integration by parts leads to

$$
\begin{aligned}
I_{2}^{I} & \leq C k\left(\frac{1}{|s|+k}+\frac{1}{|s|-k}\right) \\
& +C k\left|\int_{1 / k}^{4 R} \frac{e^{\mathrm{i}(|s|+k) r}}{|s|+k} \partial_{r}\left(f^{I}(|s| r) g^{I}(k r) \mu(r)\right)+\frac{e^{\mathrm{i}(k-|s|) r}}{k-|s|} \partial_{r}\left(f^{I I}(|s| r) g^{I}(k r) \mu(r)\right)\right| .
\end{aligned}
$$

Since $|s| \geq \lambda k$, Lemma A.1 provides the estimates

$$
\left|\partial_{r}\left(f^{I}(s r) g^{I}(k r) \mu(r)\right)\right|+\left|\partial_{r}\left(f^{I I}(s r) g^{I}(k r) \mu(r)\right)\right| \leq C\left(\frac{1}{R}+\frac{1}{k r^{2}}\right), \quad 1 / k \leq r .
$$

Combining these results, we arrive at

$$
I_{2}^{I} \leq C \frac{1}{\lambda-1} .
$$

Observing $1+(\lambda-1)^{-1} \leq 2+\left(\lambda^{2}-1\right)^{-1}$ allows us to conclude the proof.

3.3. Existence and uniqueness. Existence, uniqueness, and well-posedness of problem (2.6) has been studied in much more generality (concerning the assumption on the domain $\Omega$ ) in 12 by using different techniques.

The main goal of the estimates which we have derived in the previous sections is their application to the proof of the discrete stability for the finite element discretization and the convergence rates. However, since existence, uniqueness, and well-posedness for our model problem are simple by-products we state them in passing.

Theorem 3.8. Let $B_{R}$ be a ball of radius $R>0$. Then, there exists a constant $C(R, k)>0$ such that for all $f \in\left(H^{1}\left(B_{R}\right)\right)^{\prime}$ the unique solution $u$ of problem (2.6) satisfies

$$
\|u\|_{\mathcal{H}} \leq C(R, k)\|f\|_{H^{1}\left(B_{R}\right)^{\prime}} .
$$

Proof. The coercivity of the bilinear form $a(u, v)$ follows from the compact embedding $H^{1}\left(B_{R}\right) \stackrel{c}{\hookrightarrow} L^{2}\left(B_{R}\right)$ and (3.4b), (3.4] $)$ :

$$
\operatorname{Re} a(u, u) \geq\|u\|_{\mathcal{H}}^{2}-2 k^{2}\|u\|_{L^{2}\left(B_{R}\right)}^{2}-\operatorname{Re} \int_{\partial B_{R}}\left(T_{k} u\right) \bar{u} \geq\|u\|_{\mathcal{H}}^{2}-2 k^{2}\|u\|_{L^{2}\left(B_{R}\right)}^{2} .
$$


Next, we show uniqueness of the adjoint problem (see, e.g., [29, p. 43]):

$$
\overline{a(v, u)}=0 \quad \forall v \in H^{1}\left(B_{R}\right) \Longrightarrow u=0 .
$$

Let $u \in H^{1}\left(B_{R}\right)$ be a solution of the homogeneous adjoint problem. We choose $v=u$ and consider the imaginary part:

$$
0=\operatorname{Im} \overline{a(u, u)}=-\operatorname{Im} \overline{\int_{\partial B_{R}}\left(T_{k} u\right) \bar{u}}=\operatorname{Im} \int_{\partial B_{R}}\left(T_{k} u\right) \bar{u} .
$$

Lemma 3.3 implies $u=0$ on $\partial B_{R}$. Hence, $u \in H_{0}^{1}\left(B_{R}\right)$ and satisfies

$$
\int_{B_{R}}\langle\nabla u, \nabla \bar{v}\rangle=k^{2} \int_{B_{R}} u \bar{v} \quad \forall v \in H^{1}\left(B_{R}\right) .
$$

This means in particular that $u \in H_{0}^{1}\left(B_{R}\right)$ is an eigenfunction of $(-\Delta)^{-1}$ with eigenvalue $k^{-2}$. However, for any domain $\tilde{\Omega} \supset B_{R}$, equation (3.45) implies that the extension

$$
\tilde{u}(x):= \begin{cases}u(x) & x \in B_{R}, \\ 0 & x \notin B_{R}\end{cases}
$$

satisfies (3.45) with $B_{R}$ replaced by $\tilde{\Omega}$, i.e., $\tilde{u}$ is also an eigenfunction of $(-\Delta)^{-1}$ with eigenvalue $k^{-2}$ on any domain $\tilde{\Omega} \supset B_{R}$. A simple scaling argument shows that this is impossible.

Thus, the assertion follows from the theory of Fredholm operators (see, e.g., 29, Theorem 2.4]).

Note that the proof of Theorem 3.8 does not provide how the constant $C(R, k)$ depends on the wave number. In [12, this question has been investigated in much more generality and, hence, will not be discussed here. The Fourier analysis which we developed in Section 3.2 gives explicit bounds on this constant provided the right-hand side is in $L^{2}(\Omega)$.

Lemma 3.9. Let $\Omega$ be a bounded domain which is contained in the ball $B_{R}$ for some $R$ satisfying (3.3). For any $f \in L^{2}(\Omega)$ and $v:=N_{k} f$, there holds

$$
\|v\|_{\mathcal{H}} \leq C\|f\|_{L^{2}(\Omega)},
$$

where $C$ only depends on $k_{0}$ and $R_{0}$ (cf. (2.2), (3.3)).

Proof. The radius of the minimal ball that contains $\Omega$ is denoted by $R_{\Omega}$. If $4 k R_{\Omega}>$ 1 , the estimate

$$
\begin{gathered}
\|v\|_{L^{2}(\Omega)} \stackrel{\sqrt{3.28}}{=}\left\|v_{\mu}\right\|_{L^{2}(\Omega)} \stackrel{\sqrt{3.351, \sqrt[3.36]{,}}}{\leq}(2 \pi)^{\frac{d}{2}}\left(\max _{s \in \mathbb{R}_{\geq 0}}\left|s^{|\alpha|} \iota(s)\right|\right)\|f\|_{L^{2}(\Omega)} \\
\stackrel{\text { Lemma } 3.7(\mathrm{i})}{\leq}(2 \pi)^{\frac{d}{2}} \frac{R_{\Omega}}{k}\|f\|_{L^{2}(\Omega)}
\end{gathered}
$$

follows. The estimate

$$
\|\nabla v\|_{L^{2}(\Omega)} \leq C\left(\frac{1}{k_{0}}+R_{\Omega}\right)\|f\|_{L^{2}(\Omega)}
$$

follows by the same reasoning. If $\alpha<4 k R_{\Omega} \leq 1$, then $\left|\log k R_{\Omega}\right| \leq|\log \alpha|$. Hence, both estimates remain valid (cf. Lemma 3.7), possibly with a different constant $C$ which, in addition, depend on $\alpha$. 
3.4. An adjoint problem. The operator $N_{k}$ and the DtN operator $T_{k}$ introduced in Section 2 are associated with the outgoing radiation condition. Adopting the notation $\Omega$ and $\Omega^{+}$of Section 2 and assuming supp $f \subset \Omega$, one can define a problem with incoming radiation conditions: Find $u \in H^{1}(\Omega)$ and $u^{+} \in H_{l o c}^{1}\left(\Omega^{+}\right)$such that

$$
\begin{array}{cl}
\left(-\Delta-k^{2}\right) u=\bar{f} & \text { in } \Omega, \\
\left(-\Delta-k^{2}\right) u^{+}=0 & \text { in } \Omega^{+}, \\
u=u^{+} \text {and } \partial u / \partial n=\partial u^{+} / \partial n & \text { on } \partial \Omega, \\
\left|\frac{\partial u^{+}}{\partial r}+\mathrm{i} k u^{+}\right|=o\left(\|\mathbf{x}\|^{\frac{1-d}{2}}\right) & \|\mathbf{x}\| \rightarrow \infty .
\end{array}
$$

For $k>0$, we see that the complex conjugate $\bar{u}$ and $\overline{u^{+}}$of the solution satisfy (2.3). By uniquess, this allows us to identify the solution operator $N_{k}^{\star}: L^{2}(\Omega) \rightarrow H^{1}(\Omega)$ for the $u$-component of the solution of (3.46), namely,

$$
u=N_{k}^{\star}(f)=\overline{N_{k}(f)}=\int_{\Omega} \bar{G}_{k}(x-y) \bar{f}(y) d y .
$$

The solution component $u^{+}$is related to a Dirichlet-to-Neumann map. For the incoming radiation condition, this operator is given by $T_{k}^{\star} g:=\gamma_{1} w$, where $w \in$ $H_{\text {loc }}^{1}\left(\Omega^{+}\right)$solves:

$$
\text { Find } w \in H_{\text {loc }}^{1}\left(\Omega^{+}\right) \text {such that }\left\{\begin{array}{cl}
\left(-\Delta-k^{2}\right) w=0 & \text { in } \Omega^{+}, \\
w=g & \text { on } \partial \Omega, \\
\left|\frac{\partial w}{\partial r}+\mathrm{i} k w\right|=o\left(\|\mathbf{x}\|^{\frac{1-d}{2}}\right) & \|\mathbf{x}\| \rightarrow \infty .
\end{array}\right.
$$

Again by using $k>0$ and complex conjugation, we note (again by uniqueness) the representation $T_{k}^{\star} g=\overline{T_{k} \bar{g}}$. We employed the notation $T_{k}^{\star}$ since the operator $T_{k}^{\star}$ is the adjoint of $T_{k}$ with respect to the $L^{2}\left(\partial B_{R}\right)$ inner product in the case of a ball:

Lemma 3.10. Let $\Omega=B_{R} \subset \mathbb{R}^{d}, d \in\{1,2,3\}$. Then $\int_{\partial B_{R}} T_{k} u \bar{v}=\int_{\partial B_{R}} u \overline{T_{k}^{\star} v}$ for all $u, v \in H^{1 / 2}\left(\partial B_{R}\right)$.

Proof. We will only consider the case $d=2$. We expand $u$ and $v$ as in (3.9) with coefficients $\left(u_{\ell}\right)_{\ell \in \mathbb{Z}},\left(v_{\ell}\right)_{\ell \in \mathbb{Z}}$. For the calculations below, we assume that only finitely many coefficients $u_{\ell}, v_{\ell}$ are non-zero; the generalization to $u, v \in H^{1 / 2}\left(\partial B_{R}\right)$ then follows by a density argument.

We see immediately from (3.10) that $w_{\ell}(r)=w_{-\ell}(r)$. From the orthogonality properties of functions $\mathrm{e}^{\mathrm{i} \ell \theta}$ with the representation of $T_{k}$ in (3.10) we get

$$
\int_{\partial B_{R}} u \overline{T_{k}^{\star} v}=\int_{\partial B_{R}} u T_{k} \bar{v}=2 \pi \sum_{\ell \in \mathbb{Z}} u_{\ell} \bar{v}_{\ell} w_{-\ell}(k R)=2 \pi \sum_{\ell \in \mathbb{Z}} u_{\ell} \bar{v}_{\ell} w_{\ell}(k R)=\int_{\partial B_{R}} T_{k} u \bar{v} .
$$

\section{Stability AND CONVERGEnCE ANALYSiS}

This section is devoted to the analysis of the discrete problem (2.8) for the finitedimensional space $S \subset H^{1}(\Omega)$; we will provide conditions on $S$ under which unique solvability and quasi-optimality of (2.8) can be guaranteed. 
We employ the generalization of the theory of 33 that has been developed in [39. There, a measure of "almost invariance" 2 of the approximation space $S$ under the solution operator of an adjoint Helmholtz problem has been introduced.

Adjoint Problem: The weak formulation of problem (2.5) corresponds to the sesquilinear form $a(\cdot, \cdot)$ as in (2.6), where $\Omega$ may be chosen as a ball $B_{R}$ with sufficiently large radius $R$. The adjoint sesquilinear form $a^{\star}(\cdot, \cdot)$ is defined by (see, e.g., [29, p. 43])

$$
a^{\star}(u, v)=\overline{a(v, u)}
$$

For given $f \in L^{2}\left(B_{R}\right)$, the corresponding adjoint problem is given by finding $z \in H^{1}\left(B_{R}\right)$ such that

$$
a^{\star}(z, v)=(v, f)_{L^{2}\left(B_{R}\right)} \quad \forall v \in H^{1}\left(B_{R}\right) .
$$

Explicitly, we have

$$
a^{\star}(z, v)=\int_{B_{R}}\langle\nabla u, \nabla \bar{v}\rangle-k^{2} u \bar{v}-\int_{\partial B_{R}} u\left(\overline{T_{k} v}\right) .
$$

From Lemma 3.10 we conclude that

$$
a^{\star}(z, v)=\int_{B_{R}}\langle\nabla u, \nabla \bar{v}\rangle-k^{2} u \bar{v}-\int_{\partial B_{R}} T_{k}^{\star} u \bar{v} .
$$

The strong formulation of the adjoint problem is: Find $z$ such that

$$
-\Delta z-k^{2} z=\bar{f} \quad \text { in } B_{R}, \quad \frac{\partial z}{\partial n}=T_{k}^{\star} z \quad \text { on } \partial B_{R} .
$$

Recalling the definition of $T_{k}^{\star}$, we see that the solution $z$ of this problem is given by the solution $u$ of (3.46); the solution formula (3.47) therefore allows us to write the solution of (4.1) as

$$
z=N_{k}^{\star} f=\int_{\Omega} \overline{G_{k}}(x-y) \bar{f}(y) d y .
$$

In view of $\bar{z}=N_{k} f$ and $\|z\|_{\mathcal{H}}=\|\bar{z}\|_{\mathcal{H}}$, we obtain from Lemma 3.9 the following observation:

Lemma 4.1. Let $\Omega$ be a bounded Lipschitz domain and $k \geq k_{0}$. Then the constant

$$
\sup _{f \in L^{2}(\Omega) \backslash\{0\}} \frac{\left\|N_{k}^{\star} f\right\|_{\mathcal{H}}}{\|f\|_{L^{2}(\Omega)}}=: C_{\text {stab }}<\infty
$$

is independent of $k$ and depends solely on $\Omega$.

For the stability of the discrete problem, the following adjoint approximation property plays a crucial role:

$$
\eta(S):=\sup _{f \in L^{2}(\Omega) \backslash\{0\}} \inf _{v \in S} \frac{\left\|N_{k}^{\star} f-v\right\|_{\mathcal{H}}}{\|f\|_{L^{2}(\Omega)}} .
$$

(Note that the quantity $\eta(S)$ was denoted in [39] by $\tilde{\eta}(S)$.)

\footnotetext{
${ }^{2}$ We slightly changed the definition here and denote the new quantity by "adjoint approximation property".
} 
4.1. Discrete stability. In this section, we will prove the discrete stability in the form of an inf-sup condition.

Theorem 4.2. Let $\Omega=B_{R}$ be a ball with radius $R$ and let the assumptions of Lemma 3.3 be satisfied. Assume that the space $S$ is chosen such that

$$
k \eta(S) \leq \frac{1}{4 C_{c}},
$$

where $C_{c}$ is defined in Corollary 3.4. Then, with $C_{\text {stab }}$ defined in Lemma 4.1, the discrete inf-sup constant satisfies

$$
\inf _{u \in S} \sup _{v \in S \backslash\{0\}} \frac{|a(u, v)|}{\|u\|_{\mathcal{H}}\|v\|_{\mathcal{H}}} \geq \frac{1}{2+C_{c}^{-1}+4 k C_{\text {stab }}},
$$

and this ensures existence and uniqueness of the discrete problem (2.8).

Proof. Let $u \in S$ and set $z:=2 k^{2} N_{k}^{\star} u$. Then,

$$
\begin{aligned}
a(u, u+z) & =\left(\int_{B_{R}}\langle\nabla u, \nabla \bar{u}\rangle+k^{2}|u|^{2}-\int_{\partial B_{R}}\left(T_{k} u\right) \bar{u}\right)+a(u, z)-2 k^{2} \int_{B_{R}}|u|^{2} \\
& =\int_{B_{R}}\langle\nabla u, \nabla \bar{u}\rangle+k^{2}|u|^{2}-\int_{\partial B_{R}}\left(T_{k} u\right) \bar{u} .
\end{aligned}
$$

We derive from Lemma 3.3 that

$$
\operatorname{Re} a(u, u+z) \geq\|u\|_{\mathcal{H}}^{2} .
$$

Let $z_{S} \in S$ denote the best approximation of $z$ with respect to the $\|\cdot\|_{\mathcal{H}^{-}}$norm. Then,

$$
\begin{aligned}
\operatorname{Re} a\left(u, u+z_{S}\right) & \geq \operatorname{Re} a(u, u+z)-\left|a\left(u, z-z_{S}\right)\right| \stackrel{\text { Cor. [3.4 }}{\geq} \mid u\left\|_{\mathcal{H}}^{2}-C_{c}\right\| u\left\|_{\mathcal{H}}\right\| z-z_{S} \|_{\mathcal{H}} \\
& \geq\|u\|_{\mathcal{H}}\left(\|u\|_{\mathcal{H}}-2 k^{2} C_{c} \eta(S)\|u\|_{L^{2}\left(B_{R}\right)}\right) \geq\left(1-2 k C_{c} \eta(S)\right)\|u\|_{\mathcal{H}}^{2} .
\end{aligned}
$$

The stability of the continuous problem (cf. Lemma 4.1) implies

$$
\begin{aligned}
\left\|u+z_{S}\right\|_{\mathcal{H}} & \leq\|u\|_{\mathcal{H}}+\left\|z-z_{S}\right\|_{\mathcal{H}}+\|z\|_{\mathcal{H}} \\
& \leq\|u\|_{\mathcal{H}}+2 k^{2} \eta(S)\|u\|_{L^{2}\left(B_{R}\right)}+2 k^{2} C_{\text {stab }}\|u\|_{L^{2}\left(B_{R}\right)} \\
& \leq\left(1+2 k \eta(S)+2 k C_{\text {stab }}\right)\|u\|_{\mathcal{H}}
\end{aligned}
$$

so that

$$
\operatorname{Re} a\left(u, u+z_{S}\right) \geq \frac{1-2 C_{c} k \eta(S)}{1+2 k \eta(S)+2 k C_{\text {stab }}}\|u\|_{\mathcal{H}}\left\|u+z_{S}\right\|_{\mathcal{H}} .
$$

Therefore, in view of the assumption (4.6), we have proved

$$
\inf _{u \in S} \sup _{v \in S \backslash\{0\}} \frac{|a(u, v)|}{\|u\|_{\mathcal{H}}\|v\|_{\mathcal{H}}} \geq \frac{1}{2+C_{c}^{-1}+4 k C_{\text {stab }}} .
$$

4.2. Convergence analysis. The convergence of the finite element discretization is proved by applying the theory as developed in [39] (see also [7, 33, 40, 8, Sec. $5.7])$.

Theorem 4.3. Let the assumptions of Theorem 4.2 be satisfied. Let $u$ denote the solution of (2.6) and $u_{S}$ its Galerkin approximation (cf. (2.8)).

Then

$$
\left\|u-u_{S}\right\|_{\mathcal{H}} \leq 2 C_{c} \inf _{v \in S}\|u-v\|_{\mathcal{H}} .
$$


The $L^{2}$-error satisfies

$$
\left\|u-u_{S}\right\|_{L^{2}\left(B_{R}\right)} \leq C_{c} \eta(S)\left\|u-u_{S}\right\|_{\mathcal{H}} .
$$

Proof. In the first step, we will estimate the $L^{2}$-error by the $H^{1}$-error and employ the Aubin-Nitsche technique. The Galerkin error is denoted by $e=u-u_{S}$. We set $\psi:=N_{k}^{\star} e$ (cf. (4.3)) and denote by $\psi_{S} \in S$ the best approximation of $\psi$ with respect to the $\mathcal{H}$-norm.

The $L^{2}$-error can be estimated by

$$
\begin{aligned}
\|e\|_{L^{2}\left(B_{R}\right)}^{2} & =a(e, \psi) \leq a\left(e, \psi-\psi_{S}\right) \leq C_{c}\|e\|_{\mathcal{H}}\left\|\psi-\psi_{S}\right\|_{\mathcal{H}} \\
& \leq C_{c} \eta(S)\|e\|_{\mathcal{H}}\|e\|_{L^{2}\left(B_{R}\right)},
\end{aligned}
$$

i.e.,

$$
\|e\|_{L^{2}\left(B_{R}\right)} \leq C_{c} \eta(S)\|e\|_{\mathcal{H}} .
$$

To estimate the $\mathcal{H}$-norm of the error we proceed as follows. Note that 3.4b), 3.4k imply

$$
\operatorname{Re}\left(T_{k} u, u\right)_{L^{2}\left(\partial B_{R}\right)} \leq 0 .
$$

Hence, for any $v_{S} \in S$,

$$
\begin{aligned}
\|e\|_{\mathcal{H}}^{2} & =\operatorname{Re}(a(e, e))+\left\{\|e\|_{\mathcal{H}}^{2}-\operatorname{Re} a(e, e)\right\} \\
& =\operatorname{Re} a\left(e, u-v_{S}\right)+2 k^{2}\|e\|_{L^{2}\left(B_{R}\right)}^{2}+\operatorname{Re} \int_{\partial B_{R}}\left(T_{k} e\right) \bar{e} \\
& \stackrel{\leq 4.91,}{\leq} C_{c}\|e\|_{\mathcal{H}}\left\|u-v_{S}\right\|_{\mathcal{H}}+2 k C_{c} \eta(S)\|e\|_{\mathcal{H}}^{2} .
\end{aligned}
$$

Noting that (4.6) implies $2 k C_{c} \eta(S) \leq 1 / 2$ we arrive at the final estimate $\|e\|_{\mathcal{H}} \leq$ $2 C_{c}\left\|u-v_{S}\right\|_{\mathcal{H}}$.

\section{EXAMPLE: $h p$-FEM}

Theorems 4.2, 4.3 show quasi-optimality of arbitrary approximation spaces under the assumption (4.6) on the adjoint approximation property $\eta(S)$. However, for concrete finite element spaces, or generalizations thereof, the verification of condition (4.6) is far from trivial. The purpose of this section is two-fold: First, we show that for classical higher order FEM spaces the assumption (4.6) can be met under a relatively mild condition on the local polynomial order of the classical FEM space; in particular, we will demonstrate that for spaces consisting of piecewise polynomials of degree $p$ on quasi-uniform meshes that satisfy the side condition $p \geq c \ln k$, the key condition (4.6) is satisfied. Second, we derive conditions on the approximation space that may be easier to ascertain in practice than the condition (4.6).

In view of the fact that the circle (in 2D) and the sphere (in 3D) are relevant geometries for our theory (recall that Theorems 4.24 .3 have been shown for circles/spheres), we consider triangulations with curved elements that permit inclusion of these geometries. Before formulating the conditions on the mesh in an abstract way, we give an example of a typical construction.

Example 5.1 (Patchwise construction of FE mesh.). Let $\Omega$ denote a bounded domain. 
1. We assume that there exists a polyhedral (polygonal in $2 \mathrm{D}$ ) domain $\widetilde{\Omega}$ along with a bi-Lipschitz mapping $\chi: \widetilde{\Omega} \rightarrow \Omega$. Let $\widetilde{\mathcal{T}}^{\text {macro }}=\left\{\widetilde{K}_{i}^{\text {macro }}: 1 \leq i \leq q\right\}$ denote a conforming finite element mesh for $\widetilde{\Omega}$ consisting of simplices which are regular in the sense of $\left[13 . \widetilde{\mathcal{T}}^{\text {macro }}\right.$ is considered as a coarse partition of $\widetilde{\Omega}$, i.e., the diameters of the elements in $\widetilde{\mathcal{T}}^{\text {macro }}$ are of order 1 . We assume that the restrictions $\chi_{i}:=\left.\chi\right|_{\widetilde{K}_{i}^{\text {macro }}}$ are analytic for all $1 \leq i \leq q$.

2. The finite element mesh with step size $h$ is generated by refining the mesh $\widetilde{\mathcal{T}}^{\text {macro }}$ in a standard way (e.g., in $2 \mathrm{D}$, by connecting the midpoints of the triangle edges) and denoted by $\widetilde{\mathcal{T}}_{h}=\left\{\widetilde{K}_{i}: 1 \leq i \leq N\right\}$. The corresponding finite element mesh for $\Omega$ is then defined by $\mathcal{T}_{h}=\left\{K=\chi(\widetilde{K}): \widetilde{K} \in \widetilde{\mathcal{T}}_{h}\right\}$.

Note that, for any $K=\chi(\widetilde{K}) \in \mathcal{T}_{h}$, there exists an affine bijection $A_{K}: \widehat{K} \rightarrow \widetilde{K}$ which maps the reference element $\widehat{K}:=\left\{x \in\left(\mathbb{R}_{\geq 0}\right)^{d}: \sum_{i=1}^{d} x_{i} \leq 1\right\}$ to the simplex $\widetilde{K}$. A parametrization $F_{K}: \widehat{K} \rightarrow K$ can be chosen by $F_{K}:=R_{K} \circ A_{K}$, where $R_{K}:=\left.\chi\right|_{\widetilde{K}}$ is independent of the mesh width $h:=\max \left\{\operatorname{diam} K: K \in \mathcal{T}_{h}\right\}$.

To formulate the smoothness and scaling assumptions on $R_{K}$ and $A_{K}$ in an abstract way we have to introduce some notation first. For a function $u: \Omega \rightarrow \mathbb{R}$, $\Omega \subset \mathbb{R}^{d}$, we write

$$
\left|\nabla^{n} u(x)\right|^{2}=\sum_{\alpha \in \mathbb{N}_{0}^{d}:|\alpha|=n} \frac{n !}{\alpha !}\left|D^{\alpha} u(x)\right|^{2} .
$$

For later purposes, we recall the multinomial formula and a simple fact that follows from the Cauchy-Schwarz inequality for sums:

$$
\begin{aligned}
\frac{d^{n}}{n !} & =\sum_{\alpha \in \mathbb{N}_{0}^{d}:|\alpha|=n} \frac{1}{\alpha !}, \\
\sum_{\alpha \in \mathbb{N}_{0}^{d}:|\alpha|=n} \frac{1}{\alpha !}\left|D^{\alpha} u(x)\right| & \leq \frac{1}{n !} d^{n / 2}\left|\nabla^{n} u(x)\right| .
\end{aligned}
$$

Assumption 5.2 (quasi-uniform regular triangulation). Each element map $F_{K}$ can be written as $F_{K}=R_{K} \circ A_{K}$, where $A_{K}$ is an affine map and the maps $R_{K}$ and $A_{K}$ satisfy for constants $C_{\text {affine }}, C_{\text {metric }}, \gamma>0$ independent of $h$ :

$$
\begin{aligned}
& \left\|A_{K}^{\prime}\right\|_{L^{\infty}(\widehat{K})} \leq C_{\text {affine }} h, \quad\left\|\left(A_{K}^{\prime}\right)^{-1}\right\|_{L^{\infty}(\widehat{K})} \leq C_{\text {affine }} h^{-1}, \\
& \left\|\left(R_{K}^{\prime}\right)^{-1}\right\|_{L^{\infty}(\widetilde{K})} \leq C_{\text {metric }}, \quad\left\|\nabla^{n} R_{K}\right\|_{L^{\infty}(\widetilde{K})} \leq C_{\text {metric }} \gamma^{n} n ! \quad \forall n \in \mathbb{N}_{0} .
\end{aligned}
$$

Here, $\widetilde{K}=A_{K}(\widehat{K})$.

For meshes $\mathcal{T}_{h}$ satisfying Assumption 5.2 with element maps $F_{K}$ we denote the usual space of piecewise (mapped) polynomials by

$$
S^{p, 1}\left(\mathcal{T}_{h}\right):=\left\{u \in H^{1}(\Omega)\left|\forall K \in \mathcal{T}_{h}: u\right|_{K} \circ F_{K} \in \mathcal{P}_{p}\right\},
$$

where $\mathcal{P}_{p}$ denotes the space of polynomials of degree $p$. It is desirable to construct an approximant $I u \in S^{p, 1}\left(\mathcal{T}_{h}\right)$ of a given (sufficiently smooth) function $u$ in an elementwise fashion. The $C^{0}$-continuity of an elementwise defined approximant $I u$ is most conveniently ensured if $I u$ is defined in such a way that for every topological 
entity $E$ of the mesh (i.e., $E$ is an element $K$, a face $f$, an edge $e$, or a vertex $V$ ) the restriction $\left.(I u)\right|_{E}$ is fully determined by $\left.u\right|_{\bar{E}}$. There are many ways of realizing this construction principle. The construction employed in the present paper is based on the following concept:

Definition 5.3 (element-by-element construction). Let $\widehat{K}$ be the reference simplex in $\mathbb{R}^{d}, d \in\{2,3\}$. A polynomial $\pi$ is said to permit an element-by-element construction of polynomial degree $p$ for $u \in H^{s}(\widehat{K}), s>d / 2$, if:

(i) $\pi(V)=u(V)$ for all $d+1$ vertices $V$ of $\widehat{K}$,

(ii) for every edge $e$ of $\widehat{K}$, the restriction $\left.\pi\right|_{e} \in \mathcal{P}_{p}$ is the unique minimizer of

$$
\pi \mapsto p^{1 / 2}\|u-\pi\|_{L^{2}(e)}+\|u-\pi\|_{H_{00}^{1 / 2}(e)}
$$

under the constraint that $\pi$ satisfies (5.3); here the Sobolev norm $H_{00}^{1 / 2}$ is defined in (B.1).

(iii) (for $d=3$ ) for every face $f$ of $\widehat{K}$, the restriction $\left.\pi\right|_{f} \in \mathcal{P}_{p}$ is the unique minimizer of

$$
\pi \mapsto p\|u-\pi\|_{L^{2}(f)}+\|u-\pi\|_{H^{1}(f)}
$$

under the constraint that $\pi$ satisfies (5.3), (5.3) for all vertices and edges of the face $f$.

Remark 5.4. The conditions of Definition 5.3 are a variation of similar proposals in the literature, e.g., [14] and 21]. For example, the effective difference between the projection-based interpolation of [14 and the present construction lies in the choice of the norms employed in the minimization process in Definition 5.3. Our motivation for formulating the conditions in Definition 5.3 is that they permit us in Appendix B to construct approximation operators with optimal simultaneous approximation properties in $L^{2}$ and $H^{1}$. Previously, the literature had focused on $H^{1}$-approximation alone.

We are now in position to show that the solution $v=N_{k}^{\star} f$ can be approximated well by the FEM space $S^{p, 1}\left(\mathcal{T}_{h}\right)$ provided that $k h / p$ is sufficiently small and $p \geq$ $c \ln k$.

Theorem 5.5. Let $d \in\{1,2,3\}$ and $\Omega \subset \mathbb{R}^{d}$ be a bounded domain. Then there exist constants $C, \sigma>0$ that depend solely on the constants appearing in Assumption 5.2 such that for every $f \in L^{2}(\Omega)$ the function $v:=N_{k}^{\star} f$ satisfies

$$
\inf _{w \in S^{p, 1}\left(\mathcal{T}_{h}\right)} k\|v-w\|_{\mathcal{H}} \leq C\|f\|_{L^{2}(\Omega)}\left(1+\frac{k h}{p}\right)\left\{\frac{k h}{p}+k\left(\frac{k h}{\sigma p}\right)^{p}\right\} .
$$

Proof. We will only prove the cases $d \in\{2,3\}$. The case $d=1$ follows by similar arguments where the appeal to Theorem B.4 and Lemma C.3 is replaced with that to [41, Thm. 3.17].

We note $v=N_{k}^{\star} f=\overline{N_{k} \bar{f}}$, fix $\lambda>1$ in Lemma 3.5, and split with its aid $v=v_{H^{2}}+v_{\mathcal{A}}$ with $v_{H^{2}} \in H^{2}(\Omega)$ and $v_{\mathcal{A}}$ analytic; we have the following bounds:

$$
\left\|v_{H^{2}}\right\|_{H^{2}(\Omega)} \leq C\|f\|_{L^{2}(\Omega)}, \quad\left\|\nabla^{p} v_{\mathcal{A}}\right\|_{L^{2}(\Omega)} \leq C(\lambda k)^{p-1}\|f\|_{L^{2}(\Omega)} \quad \forall p \in \mathbb{N}_{0} .
$$

We approximate $v_{H^{2}}$ and $v_{\mathcal{A}}$ separately. Theorem B.4 and a scaling argument provides an approximant $w_{H^{2}} \in S^{p, 1}\left(\mathcal{T}_{h}\right)$ such that for every $K \in \mathcal{T}_{h}$ we have, for 
$q=0,1$,

$$
\left\|v_{H^{2}}-w_{H^{2}}\right\|_{H^{q}(K)} \leq C\left(\frac{h}{p}\right)^{2-q}\left\|v_{H^{2}}\right\|_{H^{2}(K)} \quad \forall K \in \mathcal{T}_{h} .
$$

Hence, by summation over all elements, we arrive at

$$
k\left\|v_{H^{2}}-w_{H^{2}}\right\|_{\mathcal{H}} \leq C\left(\frac{k h}{p}+\left(\frac{k h}{p}\right)^{2}\right)\|f\|_{L^{2}(\Omega)} .
$$

We now turn to the approximation of $v_{\mathcal{A}}$. Again, we construct the approximation $w_{\mathcal{A}} \in S^{p, 1}\left(\mathcal{T}_{h}\right)$ in an element-by-element fashion. We start by defining for each element $K \in \mathcal{T}_{h}$ the constant $C_{K}$ by

$$
C_{K}^{2}:=\sum_{p \in \mathbb{N}_{0}} \frac{\left\|\nabla^{p} v_{\mathcal{A}}\right\|_{L^{2}(K)}^{2}}{(2 \lambda k)^{2 p}}
$$

and we note

$$
\begin{aligned}
\left\|\nabla^{p} v_{\mathcal{A}}\right\|_{L^{2}(K)} & \leq(2 \lambda k)^{p} C_{K} \quad \forall p \in \mathbb{N}_{0}, \\
\sum_{K \in \mathcal{T}_{h}} C_{K}^{2} & \leq \frac{4}{3}\left(\frac{C}{\lambda k}\right)^{2}\|f\|_{L^{2}(\Omega)}^{2} .
\end{aligned}
$$

Let the element map for $K$ be $F_{K}=R_{K} \circ A_{K}$. Lemma C.1 gives that the function $\tilde{v}:=\left.v_{\mathcal{A}}\right|_{K} \circ R_{K}$ satisfies, for suitable constants $\tilde{C}, C$ (which depend additionally on the constants describing the analyticity of the element maps $R_{K}$ ),

$$
\left\|\nabla^{p} \tilde{v}\right\|_{L^{2}(\widetilde{K})} \leq C \tilde{C}^{p} \max \{p, k\}^{p} C_{K} \quad \forall p \in \mathbb{N}_{0} .
$$

Since $A_{K}$ is affine, the function $\hat{v}:=\left.v_{\mathcal{A}}\right|_{K} \circ F_{K}=\tilde{v} \circ A_{K}$ therefore satisfies

$$
\left\|\nabla^{p} \hat{v}\right\|_{L^{2}(\widehat{K})} \leq C h^{-d / 2} \tilde{C}^{p} h^{p} \max \{p, k\}^{p} C_{K} \quad \forall p \in \mathbb{N}_{0} .
$$

Hence, the assumptions of Lemma C.3 (with $R=1$ there) are satisfied, and we get an approximation $w$ on the element $K$ by lifting an element-by-element construction on $\widehat{K}$ to $K$ via $F_{K}$ which satisfies for $q \in\{0,1\}$,

$$
\left\|v_{\mathcal{A}}-w\right\|_{H^{q}(K)} \leq C h^{d / 2-q} h^{-d / 2} C_{K}\left\{\left(\frac{h}{h+\sigma}\right)^{p+1}+\left(\frac{k h}{\sigma p}\right)^{p+1}\right\} .
$$

Summation over all elements $K \in \mathcal{T}_{h}$ gives

$$
\begin{aligned}
\left\|v_{\mathcal{A}}-w\right\|_{\mathcal{H}}^{2} \leq\left[\left(\frac{h}{h+\sigma}\right)^{2 p}+k^{2}\left(\frac{h}{h+\sigma}\right)^{2 p+2}\right. & +\frac{k^{2}}{p^{2}}\left(\frac{k h}{\sigma p}\right)^{2 p} \\
& \left.+k^{2}\left(\frac{k h}{\sigma p}\right)^{2 p+2}\right] \sum_{K \in \mathcal{T}_{h}} C_{K}^{2} .
\end{aligned}
$$

The combination of (5.9) and (5.8) yields

$$
k\left\|v_{\mathcal{A}}-w\right\|_{\mathcal{H}} \leq C\left[\left(\frac{h}{h+\sigma}\right)^{p}\left(1+\frac{h k}{h+\sigma}\right)+k\left(\frac{k h}{\sigma p}\right)^{p}\left(\frac{1}{p}+\frac{k h}{\sigma p}\right)\right]\|f\|_{L^{2}(\Omega)} .
$$


Furthermore, we estimate using $h \leq \operatorname{diam} \Omega$ and $\sigma>0$ (independent of $h$ )

$$
\begin{aligned}
\left(\frac{h}{h+\sigma}\right)^{p}\left(1+\frac{k h}{\sigma+h}\right) & \leq C h(1+k h)\left(\frac{h}{\sigma+h}\right)^{p-1} \\
& \leq C h(1+k h) p^{-2} \leq C \frac{h}{p}\left(\frac{1}{p}+\frac{k h}{p}\right) .
\end{aligned}
$$

We therefore arrive at

$$
k\left\|v_{\mathcal{A}}-w\right\|_{\mathcal{H}} \leq C\left(\frac{1}{p}+\frac{k h}{p}\right)\left[\frac{k h}{p}+k\left(\frac{k h}{\sigma p}\right)^{p}\right]\|f\|_{L^{2}(\Omega)},
$$

which completes the proof of the theorem.

Combining Theorems $5.5,4.3$ produces the condition (1.1) for quasi-optimality of the $h p$-FEM announced in the Introduction. We extract from Theorem 5.5 that quasi-optimality of the $h$-version FEM can be achieved under the side condition that $p \geq C \log k$ :

Corollary 5.6. Let $\Omega=B_{R}$ be a ball of radius $R$ and assume (3.3), (2.2) with the additional condition $k_{0} \geq 1$ in the case $d=2$. Let Assumption 5.2 be valid. Then there exist constants $c_{1}, c_{2}>0$ independent of $k, h$, and $p$ such that (4.6) is implied by the following condition:

$$
\frac{k h}{p} \leq c_{1} \quad \text { together with } \quad p \geq c_{2} \ln k .
$$

Alternatively, the discrete stability follows from

$$
p=O(1) \text { fixed independent of } k \text { and } k h+k(k h)^{p} \leq C,
$$

which is understood as a condition on the maximal step size $h$.

Proof. Theorem [5.5 implies

$$
k \eta(S) \leq C\left(1+\frac{k h}{p}\right)\left(\frac{k h}{p}+k\left(\frac{k h}{\sigma p}\right)^{p}\right) .
$$

The right-hand side needs to be bounded by $1 / C_{c}$. It is now easy to see that we can select $c_{1}, c_{2}$ such that this can be ensured.

An easy consequence of the stability result Corollary [5.6 is:

Corollary 5.7. Let the assumptions of Corollary 5.6 be satisfied and let (5.10) or (5.11) hold. Then, the Galerkin solution $u_{S}$ exists and satisfies the error estimate

$$
\left\|u-u_{S}\right\|_{\mathcal{H}} \leq C_{c}\left(\frac{h}{p}+\left(\frac{k h}{\sigma p}\right)^{p}\right)\|f\|_{L^{2}(\Omega)} .
$$

Remark 5.8. To the best of the authors' knowledge, discrete stability in $2 \mathrm{D}$ and 3D has only been shown under much more restrictive conditions than (5.10), e.g., the condition $k^{2} h \lesssim 1$. Even in one dimension, condition (5.10) improves the stability condition $k h \lesssim 1$ that was required in [27].

Finally, we reformulate Theorem 5.5 by deriving the statement under some conditions on abstract approximation spaces that may be easier to verify than a direct proof of (4.6).

The key step in Theorem 5.5 is the ability to decompose $v=N_{k}^{\star} f$ into an analytic, but oscillatory part and an $\mathrm{H}^{2}$-regular part and to approximate each part separately. This gives rise to the definition of two types of approximation properties. 
Definition 5.9. For given $\gamma>0$ and $k>0$ let

$$
\begin{aligned}
\mathcal{H}^{\mathrm{osc}}(\gamma, k) & :=\left\{v \in H^{1}(\Omega) \mid\left\|\nabla^{p} v\right\|_{L^{2}(\Omega)} \leq(\gamma k)^{p-1} \quad \forall p \in \mathbb{N}_{0}\right\}, \\
\mathcal{H}^{H^{2}} & :=\left\{v \in H^{2}(\Omega) \mid\|v\|_{H^{2}(\Omega)} \leq 1\right\} .
\end{aligned}
$$

Let $S \subset H^{1}(\Omega)$ be the (possibly $k$-dependent) finite dimensional approximation space for the Galerkin method. The approximation properties for the oscillatory and the $H^{2}$-part are

$$
\begin{aligned}
\eta_{\mathcal{A}}(S, k, \gamma) & :=\sup _{v \in \mathcal{H}^{\circ \operatorname{sc}}(\gamma, k)} \inf _{w \in S}\|v-w\|_{\mathcal{H}}, \\
\eta_{H^{2}}(S) & :=\sup _{v \in \mathcal{H}^{H^{2}}} \inf _{w \in S}\|v-w\|_{\mathcal{H}} .
\end{aligned}
$$

The decomposition Lemma 3.5 allows us to recast $\eta(S)$ in terms of $\eta_{\mathcal{A}}(S, k, \gamma)$ and $\eta_{H^{2}}(S)$ :

Lemma 5.10. Let $\Omega \subset \mathbb{R}^{d}, d \in\{1,2,3\}$, be a bounded domain and select $\lambda>1$. Set $\gamma:=\sqrt{d} \lambda$ and define

$$
C_{H^{2}}:=\sup _{f \in L^{2}(\Omega)} \frac{\left\|v_{H^{2}}\right\|_{\mathcal{H}}}{\|f\|_{L^{2}(\Omega)}}, \quad C_{\mathcal{A}}:=\sup _{f \in L^{2}(\Omega)} \sup _{p \in \mathbb{N}_{0}} \frac{\left\|\nabla^{p} v_{\mathcal{A}}\right\|_{L^{2}(\Omega)}}{(\gamma k)^{p-1}\|f\|_{L^{2}(\Omega)}},
$$

where, for each $f \in L^{2}(\Omega)$ we employ the $\lambda$-dependent decomposition $N_{k}^{\star} f=v_{H^{2}}+$ $v_{\mathcal{A}}$ according to Lemma 3.5. Let $S \subset H^{1}(\Omega)$ be a finite dimensional approximation space. Then, the adjoint approximability $\eta(S)$ is bounded by

$$
\eta(S) \leq C_{\mathcal{A}} \eta_{\mathcal{A}}(S, k, \gamma)+C_{H^{2}} \eta_{H^{2}}(S) .
$$

Before proving this statement, we stress that the scaling in Definition 5.9 has been chosen such that, according to Lemma 3.5, the constants $C_{\mathcal{A}}$ and $C_{H^{2}}$ are bounded uniformly in $k$.

Proof. For $f \in L^{2}(\Omega)$, we employ the splitting $v=N_{k}^{\star} f=v_{H^{2}}+v_{\mathcal{A}}$ as in Lemma 3.5 for the selected $\lambda>1$. We set

$$
\widetilde{v_{H^{2}}}:=\left\{\begin{array}{cl}
0 & \text { if } f=0, \\
\frac{v_{H^{2}}}{C_{H^{2}}\|f\|_{L^{2}(\Omega)}} & \text { if } f \neq 0,
\end{array} \quad \text { and } \quad \widetilde{v_{\mathcal{A}}}:=\left\{\begin{array}{cl}
0 & \text { if } f=0, \\
\frac{v_{\mathcal{A}}}{C_{\mathcal{A}}\|f\|_{L^{2}(\Omega)}} & \text { if } f \neq 0,
\end{array}\right.\right.
$$

and note $\widetilde{v_{H^{2}}} \in \mathcal{H}^{H^{2}}$ and $\widetilde{v_{\mathcal{A}}} \in \mathcal{H}^{\text {osc }}(\gamma, k)$. Then,

$$
\begin{aligned}
\eta(S)= & \sup _{f \in L^{2}(\Omega) \backslash\{0\}} \inf _{w \in S} \frac{\left\|v_{\mathcal{A}}+v_{H_{2}}-w\right\|_{\mathcal{H}}}{\|f\|_{L^{2}(\Omega)}} \\
\leq & \sup _{f \in L^{2}(\Omega) \backslash\{0\}} \inf _{w \in S} \frac{\left\|v_{\mathcal{A}}-w\right\|_{\mathcal{H}}}{\|f\|_{L^{2}(\Omega)}}+\sup _{f \in L^{2}(\Omega) \backslash\{0\}} \inf _{w \in S} \frac{\left\|v_{H_{2}}-w\right\|_{\mathcal{H}}}{\|f\|_{L^{2}(\Omega)}} \\
\leq & \sup _{f \in L^{2}(\Omega) \backslash\{0\}} \frac{\left\|v_{\mathcal{A}}\right\|_{\mathcal{H}} /\left\|\widetilde{v_{\mathcal{A}}}\right\|_{\mathcal{H}}}{\|f\|_{L^{2}(\Omega)}} \inf _{w \in S}\left\|\widetilde{v_{\mathcal{A}}}-w\right\|_{\mathcal{H}} \\
& \quad+\sup _{f \in L^{2}(\Omega) \backslash\{0\}} \frac{\left\|v_{H^{2}}\right\|_{\mathcal{H}} /\left\|\widetilde{v_{H^{2}}}\right\|_{\mathcal{H}}}{\|f\|_{L^{2}(\Omega)}} \inf _{w \in S}\left\|\widetilde{v_{H_{2}}}-w\right\|_{\mathcal{H}} \\
\leq & C_{\mathcal{A}} \eta_{\mathcal{A}}(S)+C_{H^{2}} \eta_{H^{2}}(S) .
\end{aligned}
$$




\section{Appendix A. Estimate of Bessel functions}

In this appendix we derive some estimates for the Hankel and Bessel functions that are used in Subsection 3.2. First, we will consider the case of large arguments $z>1$ and then the case $0<z \leq 1$.

Case 1: $z=k r>1$.

From [1, 9.2.5-9.2.16], we conclude that the Hankel functions $H_{\ell}^{(1)}$ and Bessel functions $J_{\ell}, \ell \in \mathbb{N}_{0}$, can be written in the form

$$
\begin{aligned}
& \left.J_{\ell}(z) \stackrel{11}{=} \sqrt[9.5]\right]{=} \sqrt{\frac{2}{\pi z}}\left(P_{\ell}(z) \cos \chi-Q_{\ell}(z) \sin \chi\right), \\
& \left.H_{\ell}^{(1)}(z) \stackrel{11}{=} \sqrt[9.2 .7]\right]{\frac{2}{\pi z}}\left(P_{\ell}(z)+\mathrm{i} Q_{\ell}(z)\right) \mathrm{e}^{\mathrm{i} \chi},
\end{aligned}
$$

where $\chi:=z-\pi / 4$. The functions $P_{\ell}, Q_{\ell}$ have the following property: Upon defining

$$
\begin{aligned}
P_{\ell, m}(z) & :=\sum_{k=0}^{m} \frac{\beta_{\ell, 2 k}}{z^{2 k}}, \\
Q_{\ell, m}(z) & =-\mathrm{i} \sum_{k=0}^{m} \frac{\beta_{\ell, 2 k+1}}{z^{2 k+1}}
\end{aligned}
$$

with

there holds

$$
\beta_{\ell, k}:=\frac{\mathrm{i}^{k} \gamma_{\ell, k}}{2^{3 k} k !} \text { and } \gamma_{\ell, m} \text { as in (3.12) }
$$

$$
\forall z>0 \quad \forall m>\frac{\ell}{2}-\frac{1}{4}\left\{\begin{array}{l}
\left|\left(P_{\ell}-P_{\ell, m-1}\right)(z)\right| \leq \frac{\left|\gamma_{\ell, 2 m}\right|}{2^{6 m}(2 m) !} \frac{1}{z^{2 m}}, \\
\left|\left(Q_{\ell}-Q_{\ell, m-1}\right)(z)\right| \leq \frac{\gamma_{\ell, 2 m+1}}{2^{6 m+2}(2 m+1) !} \frac{1}{z^{2 m+1}} .
\end{array}\right.
$$

Note that in Subsection 3.2 the order $\ell$ is always small, i.e., $\ell \in\{0,1\}$ and, hence, we do not analyze the dependence of the constants on $\ell$ in the following estimates.

We conclude that

$$
\forall z \geq 1:\left|P_{\ell}(z)\right| \leq\left|P_{\ell,\left\lceil\frac{\ell}{2}\right\rceil-1}(z)\right|+\frac{\left|\gamma_{\ell, 2\left\lceil\frac{\ell}{2}\right\rceil}\right|}{2^{6\left\lceil\frac{\ell}{2}\right\rceil}\left(2\left\lceil\frac{\ell}{2}\right\rceil\right) !} \frac{1}{z^{2\left\lceil\frac{\ell}{2}\right\rceil}} \leq C,
$$

and similarly,

$$
\forall z \geq 1: \quad\left|Q_{\ell}(z)\right| \leq \frac{C}{z}, \quad\left|P_{\ell}^{\prime}(z)\right| \leq \frac{C}{z^{3}}, \quad\left|Q_{\ell}^{\prime}(z)\right| \leq \frac{C}{z^{2}} .
$$

Hence, for $f \in\left\{J_{\ell}, H_{\ell}^{(1)}\right\}, \ell \in \mathbb{N}_{0}$, there holds

$$
\forall z \geq 1: \quad|f(z)| \leq \frac{C}{\sqrt{z}},
$$

and the combination with $\left|J_{\ell}(z)\right| \stackrel{10}{\leq} C$ for all $z \geq 0$ yields

$$
\forall z \geq 0: \quad\left|J_{\ell}(z)\right| \leq C \sqrt{\frac{1}{1+z}} .
$$


We need an estimate of the derivative at the argument $z=k r$ for $z \geq 1$. The derivative of (A.1b) can be written in the form

$$
\begin{aligned}
\frac{d}{d r} H_{0}^{(1)}(k r) \stackrel{[1]}{\stackrel{9.2 .7]}{=}} C \mathrm{e}^{\mathrm{i} k r} \sqrt{\frac{1}{k r}} \frac{d}{d r}\left(P_{0}(k r)+\mathrm{i} Q_{0}(k r)\right) \\
+C\left(P_{0}(k r)+\mathrm{i} Q_{0}(k r)\right) \frac{d}{d r}\left(\mathrm{e}^{\mathrm{i} k r} \sqrt{\frac{1}{k r}}\right) .
\end{aligned}
$$

The combination of (A.4) and (A.2) leads to

$$
\left|\frac{d}{d r} H_{0}^{(1)}(k r)\right| \leq C\left(\frac{1}{r \sqrt{k r}}+\sqrt{\frac{k}{r}}\right) .
$$

We also need an estimate of $\partial_{r}\left(\mathrm{e}^{-\mathrm{i} k r} H_{0}^{(1)}(k r)\right)$. Employing (A.1b) we obtain

$$
\frac{d}{d r}\left(\mathrm{e}^{-\mathrm{i} k r} H_{0}^{(1)}(k r)\right)=\sqrt{\frac{2}{\pi}} e^{-\pi / 4} \frac{d}{d r}\left(\sqrt{\frac{1}{k r}}\left(P_{0}(k r)+\mathrm{i} Q_{0}(k r)\right)\right) .
$$

Thus, for $k r \geq 1$, we get

$$
\left|\partial_{r}\left(\mathrm{e}^{-\mathrm{i} k r} H_{0}^{(1)}(k r)\right)\right| \leq \frac{C}{r \sqrt{k r}} .
$$

An estimate of the second derivative of $H_{0}^{(1)}$ is derived by using [1, 9.1.27, 9.1.28]:

$$
\left|\frac{d^{2}}{d r^{2}} H_{0}^{(1)}(k r)\right|=k^{2}\left|-H_{0}^{(1)}(k r)+\frac{H_{1}^{(1)}(k r)}{k r}\right| \stackrel{\text { A.31 })}{\leq} C k \sqrt{\frac{k}{r}} .
$$

Case 2: $z=k r \in(0,1)$.

To estimate $H_{0}^{(1)}(z)$ in the range $(0,1)$ we employ

$$
H_{0}^{(1)}(z)=J_{0}(z)+i Y_{0}(z)
$$

and use for $Y_{0}(z)$ the expansion

$$
Y_{0}(z)=\frac{2}{\pi}\left(\log \frac{z}{2}\right) J_{0}(z)-\frac{2}{\pi} \sum_{k=0}^{\infty} \psi(k+1) \frac{\left(-\frac{z^{2}}{4}\right)^{k}}{(k !)^{2}},
$$

where

$$
\psi(n):=-\gamma+\sum_{k=1}^{n-1} k^{-1} \text { and } \quad \gamma:=0.57721566 \ldots \text { is Euler's constant. }
$$

For $0 \leq z \leq 1$, we have

$$
\left|Y_{0}(z)\right| \leq \frac{2}{\pi}\left|\log \frac{z}{2}\right|+\frac{2}{\pi} \sum_{k=0}^{\infty} \frac{\psi(k+1)}{4^{k}(k !)^{2}} .
$$

Furthermore,

$$
|\psi(k+1)| \leq \gamma+1+\sum_{s=2}^{k} \frac{1}{s} \leq \gamma+1+\int_{1}^{k} \frac{1}{x} d x=\gamma+1+\log k=: \gamma^{\prime}+\log k .
$$


Thus, for $0 \leq z \leq 1$, we have

$$
\left|Y_{0}(z)\right| \leq \frac{2}{\pi}\left|\log \frac{z}{2}\right|+\frac{2}{\pi}\left(\gamma+\sum_{k=1}^{\infty} \frac{\gamma^{\prime}+\log k}{4^{k}(k !)^{2}}\right)
$$

Since $\frac{\gamma^{\prime}+\log k}{4^{k} k !} \leq 1$ we get

$$
\left|Y_{0}(z)\right| \leq \frac{2}{\pi}|\log z|+C
$$

This leads to the estimate:

$$
\forall z \in] 0,1]: \quad\left|H_{0}^{(1)}(z)\right| \leq \frac{2}{\pi}|\log z|+C .
$$

The combination with A.3alinally results in

$$
\left|H_{0}^{(1)}(z)\right| \leq \min \left\{\frac{2}{\pi}|\log z|+C, \frac{C}{\sqrt{z}}\right\} .
$$

We will need further estimates of $J_{1}$ and $\partial_{r} H_{0}^{(1)}$. From [1, 9.1.60], we conclude that

$$
\forall z \geq 0: \quad J_{1}(z) \leq 1 / \sqrt{2} .
$$

For the derivative of $Y_{0}$, we obtain (by using $J_{0}^{\prime}=-J_{1}$ )

$$
Y_{0}^{\prime}(z)=\frac{2}{\pi}\left(\frac{J_{0}(z)}{z}-J_{1}(z) \log \frac{z}{2}\right)+\frac{z}{\pi} \sum_{k=0}^{\infty} \psi(k+2) \frac{\left(-\frac{z^{2}}{4}\right)^{k}}{k !(k+1) !} .
$$

For $0 \leq z \leq 1$, we obtain

$$
\frac{z}{\pi} \sum_{k=0}^{\infty} \psi(k+2) \frac{\left(-\frac{z^{2}}{4}\right)^{k}}{k !(k+1) !} \leq \frac{z}{\pi} \sum_{k=0}^{\infty} \frac{\gamma^{\prime}+\log (k+1)}{k ! 4^{k}(k+1) !} \leq \frac{z}{\pi} \sum_{k=0}^{\infty} \frac{1}{k !}=C z .
$$

Now,

$$
\left|J_{1}(z)\right| \stackrel{[1]}{\stackrel{9.1 .62]}{\leq}} z / 2
$$

and we get

$$
Y_{0}^{\prime}(z) \leq \frac{2}{\pi}\left(z^{-1}+\frac{z}{2} \log \frac{z}{2}+\frac{\mathrm{e} z}{2}\right) \leq \frac{2}{\pi z}+C .
$$

Hence, for $0 \leq r \leq 1 / k$, we arrive at

$$
\left|\partial_{r} H_{0}^{(1)}(k r)\right|=k\left(\left|J_{0}^{\prime}(k r)\right|+\left|Y_{0}^{\prime}(k r)\right|\right) \leq \frac{2}{\pi r}+C k+\frac{k^{2} r}{2} \leq C\left(\frac{1}{r}+\frac{k^{2} r}{2}\right) .
$$

In addition, we need some weighted estimates for second order derivatives of $H_{0}^{(1)}$. From (A.9) we obtain

$\partial_{r}\left(r \partial_{r} Y_{0}(k r)\right)=\frac{2 k}{\pi}\left(-2 J_{1}(k r)-k r \log \frac{k r}{2} J_{0}(k r)+k r \sum_{k=0}^{\infty} \psi(k+2) \frac{\left(-\frac{(k r)^{2}}{4}\right)^{k}}{(k !)^{2}}\right)$.

This leads to the estimate, for $0<z \leq 1$,

Note that

$$
\left|\partial_{r}\left(r \partial_{r} Y_{0}(k r)\right)\right| \leq \frac{2}{\pi} k^{2} r\left(C+\left|\log \frac{k r}{2}\right|\right) .
$$

$$
\partial_{r}\left(r \partial_{r} H_{0}^{(1)}(k r)\right)=-r k^{2} J_{0}(k r)+\mathrm{i} \partial_{r}\left(r \partial_{r} Y_{0}(k r)\right),
$$


and, hence,

$$
\forall 0 \leq k r \leq 1: \quad\left|\partial_{r}\left(r \partial_{r} H_{0}^{(1)}(k r)\right)\right| \leq \frac{2}{\pi} k^{2} r\left(C+\left|\log \frac{k r}{2}\right|\right) .
$$

We finally state a lemma required for the proof of Lemma 3.7

Lemma A.1. Let $|s| \geq k$ and $k \geq k_{0}>0$. Then

$$
r J_{0}(s r) H_{0}^{(1)}(k r)=\frac{2}{\pi \sqrt{k|s|}}\left\{\mathrm{e}^{\mathrm{i}(|s|+k) r} f^{I}(|s| r)+\mathrm{e}^{\mathrm{i}(-|s|+k) r} f^{I I}(|s| r)\right\} g^{I}(k r),
$$

where the functions $f^{I}, f^{I I}, g^{I}$ satisfy for $r \geq 1$ and $a C>0$ depending solely on $k_{0}$ :

$$
\begin{aligned}
\left|f^{I}(r)\right|+\left|f^{I I}(r)\right|+\left|g^{I}(r)\right| & \leq C, \\
r^{2}\left(\left|\frac{d}{d r} f^{I}(r)\right|+\left|\frac{d}{d r} f^{I I}(r)\right|+\left|\frac{d}{d r} g^{I}(r)\right|\right) & \leq C .
\end{aligned}
$$

Proof. By symmetry of $J_{0}$, we may assume $s>0$. Formulas A.1伍, A.1b imply the stated representation with $f^{I}(s r)=\frac{1}{2}\left(P_{0}(s r)+\mathrm{i} Q_{0}(s r)\right) \mathrm{e}^{-\mathrm{i} \pi / 4}, f^{I I}(s r)=$ $\frac{1}{2}\left(P_{0}(s r)-\mathrm{i} Q_{0}(s r)\right) \mathrm{e}^{\mathrm{i} \pi / 4}$, and $g^{I}(k r)=\left(P_{0}(k r)+\mathrm{i} Q_{0}(k r)\right) \mathrm{e}^{\mathrm{i} \pi / 4}$. The estimates for $f^{I}, f^{I I}, g^{I}$ now follow from the bounds for $P_{0}, Q_{0}, P_{0}^{\prime}, Q_{0}^{\prime}$ given in A.2国, (A.2b).

\section{Appendix B. Approximation By $h p$-Finite elements. \\ Case I: Finite REgularity}

The purpose of the present section is the proof of Theorem B.4 which constructs a polynomial approximation to a function $u \in H^{s}, s>d / 2$, in an element-byelement fashion (see Definition 5.3). The novelty of the present construction over existing operators such as those of [3, 14, 34] is that we obtain optimal rates (in p) simultaneously in the $H^{1}$-norm and the $L^{2}$-norm. Closely related results can be found in the recent paper [21, where similar duality arguments are employed to obtain estimates in $L^{2}$.

B.1. Lifting operators. In the $p$-FEM, globally continuous, piecewise polynomial approximations to a function $u$ are typically constructed in two steps: in a first step, discontinuous approximations are constructed element by element. In a second step, the jumps across the element interfaces are corrected by suitable lifting operators. The construction of these lifting operators is the purpose of the present section; the ensuing Section B.2 is devoted to the analysis of polynomial approximation.

Before proceeding we recall the definition of the Sobolev space $H_{00}^{1 / 2}(\Omega)$. If $\Omega$ is an edge or a face of a triangle or a tetrahedron, then the Sobolev norm $\|\cdot\|_{H_{00}^{1 / 2}(\Omega)}$ is defined by

$$
\|u\|_{H_{00}^{1 / 2}(\Omega)}^{2}:=\|u\|_{H^{1 / 2}(\Omega)}^{2}+\left\|\frac{u}{\sqrt{\operatorname{dist}(\cdot, \partial \Omega)}}\right\|_{L^{2}(\Omega)}^{2},
$$

and the space $H_{00}^{1 / 2}(\Omega)$ is the completion of $C_{0}^{\infty}(\Omega)$ under this norm.

Lemma B.1. Let $\widehat{K}^{2 D}$ be the reference triangle in $2 D$. Vertex and edge lifting operators can be constructed with the following properties: 
(1) For each vertex $V$ of $\widehat{K}^{2 D}$ there exists a polynomial $L_{V, p} \in \mathcal{P}_{p}$ that attains the value 1 at the vertex $V$ and vanishes on the edge of $\widehat{K}^{2 D}$ opposite to $V$. Additionally, for every $s \geq 0$, there exists $C_{s}>0$ such that $\left\|L_{V, p}\right\|_{H^{s}\left(\widehat{K}^{2 D}\right)} \leq C_{s} p^{-1+s}$.

(2) For every edge e of $\widehat{K}^{2 D}$ there exists a bounded linear operator $\pi_{e}: H_{00}^{1 / 2}(e)$ $\rightarrow H^{1}\left(\widehat{K}^{2 D}\right)$ with the following properties:

(a) $\forall u \in \mathcal{P}_{p} \cap H_{00}^{1 / 2}(e): \pi_{e} u \in \mathcal{P}_{p}$,

(b) $\forall u \in H_{00}^{1 / 2}(e):\left.\pi_{e} u\right|_{\partial \widehat{K}^{2 D} \backslash e}=0$,

(c) $\forall u \in H_{00}^{1 / 2}(e): p\left\|\pi_{e} u\right\|_{L^{2}\left(\widehat{K}^{2 D}\right)}+\left\|\pi_{e} u\right\|_{H^{1}\left(\widehat{K}^{2 D}\right)}$

$$
\leq C\left(\|u\|_{H_{00}^{1 / 2}(e)}+p^{1 / 2}\|u\|_{L^{2}(e)}\right) \text {. }
$$

Proof. Let $\widehat{K}^{2 D}=\{(x, y) \mid 0<x<1,0<y<1-x\}$. The vertex function $L_{V, p}$ for the vertex $V=(0,0)$ is defined as $L_{V, p}(x, y)=(1-(x+y))^{p}$. A simple calculation then shows the result. The functions $L_{V, p}$ for the remaining 2 vertices are obtained by suitable affine transformations.

For the edge lifting, let $e$ be the edge $e=\{(x, 0) \mid 0<x<1\}$. By 3 there exists a bounded linear operator $E: H_{00}^{1 / 2}(e) \rightarrow H^{1}\left(\widehat{K}^{2 D}\right)$ with the following properties: $\left.E u\right|_{e}=u,\left.E u\right|_{\partial \widehat{K}^{2 D} \backslash e}=0$, and $E u \in \mathcal{P}_{p}$ if $u \in \mathcal{P}_{p} \cap H_{00}^{1 / 2}(e)$. Introduce the auxiliary operator $(G u)(x, y):=(1-y)^{p}(E u)(x, y)$. By [31, Lemma B.5], we have

$$
\begin{aligned}
p\|G u\|_{L^{2}\left(\widehat{K}^{2 D}\right)}+\|G u\|_{H^{1}\left(\widehat{K}^{2 D}\right)} & \leq C\left(|E u|_{H^{1}\left(\widehat{K}^{2 D}\right)}+p^{1 / 2}\|u\|_{L^{2}(e)}\right) \\
& \leq C\left(\|u\|_{H_{00}^{1 / 2}(e)}+p^{1 / 2}\|u\|_{L^{2}(e)}\right) .
\end{aligned}
$$

Denote by $\Pi_{p}^{H^{1}}: H_{0}^{1}\left(\widehat{K}^{2 D}\right) \rightarrow H_{0}^{1}\left(\widehat{K}^{2 D}\right) \cap \mathcal{P}_{p}$ the $H^{1}$-projection and set $\pi_{e} u:=$ $E u+\Pi_{p}^{H^{1}}(G u-E u)$. Then by the stability of $\Pi_{p}^{H^{1}}$ and $E$ we have

$$
\begin{aligned}
\left\|\pi_{e} u\right\|_{H^{1}\left(\widehat{K}^{2 D}\right)} & \leq\|G u\|_{H^{1}\left(\widehat{K}^{2 D}\right)}+2\|G u-E u\|_{H^{1}\left(\widehat{K}^{2 D}\right)} \\
& \leq C\left(\|u\|_{H_{00}^{1 / 2}(e)}+p^{1 / 2}\|u\|_{L^{2}(e)}\right)
\end{aligned}
$$

which is the desired $H^{1}$-stability result. For the $L^{2}$-bound, we use a duality argument as in [21]:

$\left\|G u-E u-\Pi_{p}^{H^{1}}(G u-E u)\right\|_{L^{2}\left(\widehat{K}^{2 D}\right)} \leq C p^{-1}\left\|(G u-E u)-\Pi_{p}^{H^{1}}(G u-E u)\right\|_{H^{1}\left(\widehat{K}^{2 D}\right)}$.

The $H^{1}$-stability of $\Pi_{p}^{H^{1}}$ together with stability properties of $E$ and $G$ produce the desired $L^{2}$-bound.

Lemma B.2. Let $\widehat{K}^{3 D}$ be the reference tetrahedron in $3 D$. Vertex, edge, and face lifting operators can be constructed with the following properties:

(i) For each vertex $V$ of $\widehat{K}^{3 D}$ there exists a polynomial $L_{V, p} \in \mathcal{P}_{p}$ that attains the value 1 at the vertex $V$ and vanishes on the face opposite $V$. Additionally, for every $s \geq 0$ there exists $C_{s}>0$ such that $\left\|L_{V, p}\right\|_{H^{s}\left(\widehat{K}^{3 D}\right)} \leq$ $C_{s} p^{-3 / 2+s}$.

(ii) For every edge e of $\widehat{K}^{3 D}$ there exists a bounded linear operator $\pi_{e}: H_{00}^{1 / 2}(e)$ $\rightarrow H^{1}\left(\widehat{K}^{3 D}\right)$ with the following properties:

(a) $\pi_{e} u \in \mathcal{P}_{p}$ if $u \in \mathcal{P}_{p} \cap H_{00}^{1 / 2}(e)$, 
(b) $\left.\left(\pi_{e} u\right)\right|_{f}=0$ for the two faces $f$ with $\bar{f} \cap e=\emptyset$,

(c) for the two faces $f$ adjacent to e (i.e., $\bar{f} \cap e=e)$,

$$
\begin{aligned}
p\left\|\pi_{e} u\right\|_{L^{2}(f)}+\left\|\pi_{e} u\right\|_{H^{1}(f)} & \leq C\|u\|_{H_{00}^{1 / 2}(e)}+p^{1 / 2}\|u\|_{L^{2}(e)}, \\
\left\|\pi_{e} u\right\|_{H^{1 / 2}\left(\partial \widehat{K}^{3 D}\right)} & \leq C\left(p^{-1 / 2}\|u\|_{H_{00}^{1 / 2}(e)}+\|u\|_{L^{2}(e)}\right), \\
p\left\|\pi_{e} u\right\|_{L^{2}\left(\widehat{K}^{3 D}\right)}+\left\|\pi_{e} u\right\|_{H^{1}\left(\widehat{K}^{3 D}\right)} & \leq C\left(p^{-1 / 2}\|u\|_{H_{00}^{1 / 2}(e)}+\|u\|_{L^{2}(e)}\right) .
\end{aligned}
$$

(iii) For every face $f$ of $\widehat{K}^{3 D}$ there exists a bounded linear operator $\pi_{f}: H_{00}^{1 / 2}(f)$ $\rightarrow H^{1}\left(\widehat{K}^{3 D}\right)$ with the following properties:

$$
\begin{aligned}
& \text { (a) } \pi_{f} u \in \mathcal{P}_{p} \text { if } u \in \mathcal{P}_{p} \cap H_{00}^{1 / 2}(f), \\
& \text { (b) }\left.\left(\pi_{e} u\right)\right|_{f^{\prime}}=0 \text { for the faces } f^{\prime} \neq f, \\
& p\left\|\pi_{f} u\right\|_{L^{2}\left(\widehat{K}^{3 D}\right)}+\left\|\pi_{f} u\right\|_{H^{1}\left(\widehat{K}^{3 D}\right)} \leq C\left(\|u\|_{H_{00}^{1 / 2}(f)}+p^{1 / 2}\|u\|_{L^{2}(f)}\right) .
\end{aligned}
$$

Proof. We take the reference tetrahedron $\widehat{K}^{3 D}$ to be $\widehat{K}^{3 D}=\{(x, y, z) \mid 0<x<$ $1,0<y<1-x, 0<z<1-x-y\}$.

Proof of (i). For the vertex $V=(0,0,0)$ we select $L_{V, p}(x, y, z):=(1-(x+y+$ $z))^{p}$. A calculation shows that $L_{V, p}$ has the desired properties. The functions $L_{V, p}$ for the remaining 3 vertices are obtained by affine transformations.

Proof of (iii). [34, Lemma 8] exhibits a bounded linear operator $F: H_{00}^{1 / 2}(f) \rightarrow$ $H^{1}\left(\widehat{K}^{3 D}\right)$ with the additional property that $F u \in \mathcal{P}_{p}$ if $u \in \mathcal{P}_{p} \cap H_{00}^{1 / 2}(f)$. Without loss of generality, let $f=\partial \widehat{K}^{3 D} \cap\{z=0\}$. Define the auxiliary operator $(G u)(x, y, z):=(1-z)^{p}(F u)(x, y, z)$. This operator satisfies (see [31, Lemma B.5] where the analogous arguments are worked out in the $2 \mathrm{D}$ setting)

$$
\begin{aligned}
p\|G u\|_{L^{2}\left(\widehat{K}^{3 D}\right)}+\|G u\|_{H^{1}\left(\widehat{K}^{3 D}\right)} & \leq C\left(|F u|_{H^{1}\left(\widehat{K}^{3 D}\right)}+p^{1 / 2}\|F u\|_{L^{2}(f)}\right) \\
& \leq C\|u\|_{H_{00}^{1 / 2}(f)}+p^{1 / 2}\|u\|_{L^{2}(f)} .
\end{aligned}
$$

Again, letting $\Pi_{p}^{H^{1}}: H_{0}^{1}\left(\widehat{K}^{3 D}\right) \rightarrow H_{0}^{1}\left(\widehat{K}^{3 D}\right) \cap \mathcal{P}_{p}$ be the $H^{1}$-projection, we can set $\pi_{f} u:=F u+\Pi_{p}^{H^{1}}(G u-F u)$. The desired properties of $\pi_{f}$ are then seen in exactly the same way as in the 2D case of Lemma B.1

Proof of (ii). Set $f_{e, 1}=\partial \widehat{K}^{3 D} \cap\{z=0\}$ and $f_{e, 2}=\partial \widehat{K}^{3 D} \cap\{1-x-y-z=0\}$. The edge shared by the faces $f_{e, 1}$ and $f_{e, 2}$ is $e=\{(x, 1-x, 0) \mid 0<x<1\}$. By Lemma B.1 a function $u \in H_{00}^{1 / 2}(e)$ can be lifted to a function $E u \in H^{1}\left(f_{e, 1}\right)$ such that $\left.E u\right|_{\partial f_{e, 1} \backslash e}=0$ and

$$
p\|E u\|_{L^{2}\left(f_{e, 1}\right)}+\|E u\|_{H^{1}\left(f_{e, 1}\right)} \leq C\left(\|u\|_{H_{00}^{1 / 2}(e)}+p^{1 / 2}\|u\|_{L^{2}(e)}\right) .
$$

Additionally, if $u \in \mathcal{P}_{p}$, then $E u \in \mathcal{P}_{p}$. Since the same lifting can be done for the face $f_{e, 2}$, we can find a function, again denoted $E u \in H^{1}\left(\partial \widehat{K}^{3 D}\right)$, that vanishes on $\partial \widehat{K}^{3 D} \backslash\left(f_{e, 1} \cup f_{e, 2} \cup e\right)$, such that $p\|E u\|_{L^{2}\left(\partial \widehat{K}^{3 D}\right)}+\|E u\|_{H^{1}\left(\partial \widehat{K}^{3 D}\right)} \leq$ $C\left(\|u\|_{H_{00}^{1 / 2}(e)}+p^{1 / 2}\|u\|_{L^{2}(e)}\right)$. Additionally, $E u$ is a piecewise polynomial of degree $p$ if $u \in \mathcal{P}_{p}$. An interpolation inequality gives

$$
\begin{aligned}
\|E u\|_{H^{1 / 2}\left(\partial \widehat{K}^{3 D}\right)} & \leq C\|E u\|_{L^{2}\left(\partial \widehat{K}^{3 D}\right)}^{1 / 2}\|E u\|_{H^{1}\left(\partial \widehat{K}^{3 D}\right)}^{1 / 2} \\
& \leq C p^{-1 / 2}\left(\|u\|_{H_{00}^{1 / 2}\left(\partial \widehat{K}^{3 D}\right)}+p^{1 / 2}\|u\|_{L^{2}\left(\partial \widehat{K}^{3 D}\right)}\right) .
\end{aligned}
$$


For this function $E u$, [34, Lemma 8] provides a lifting $F u \in H^{1}\left(\widehat{K}^{3 D}\right)$ with $\|F u\|_{H^{1}\left(\widehat{K}^{3 D}\right)} \leq C\|E u\|_{H^{1 / 2}\left(\widehat{K}^{3 D}\right)}$. To get a better $L^{2}$-bound, we introduce the distance functions $d_{1}(\cdot):=\operatorname{dist}\left(\cdot, f_{e, 1}\right)$ and $d_{2}(\cdot):=\operatorname{dist}\left(\cdot, f_{e, 2}\right)$ as well as $d(\cdot):=$ $\operatorname{dist}\left(\cdot, f_{e, 1} \cup f_{e, 2}\right)=\min \left\{d_{1}(\cdot), d_{2}(\cdot)\right\}$ and set $w:=(1-d)^{p}$. Define $G u:=w F u$. Then $\left.(G u)\right|_{\partial \widehat{K}^{3 D}}=\left.(F u)\right|_{\partial \widehat{K}^{3 D}}$ since $\left.w\right|_{f_{e, 1} \cup f_{e, 2}} \equiv 1$ and $\left.F u\right|_{\partial \widehat{K}^{3 D} \backslash\left(f_{e, 1} \cup f_{e, 2}\right)}=0$. Additionally, $G u \in H^{1}\left(\widehat{K}^{3 D}\right)$ since $w$ is Lipschitz continuous. Furthermore, we have

(B.2) $p\|G u\|_{L^{2}\left(\widehat{K}^{3 D}\right)}+\|G u\|_{H^{1}\left(\widehat{K}^{3 D}\right)} \leq C\left(\|F u\|_{H^{1}\left(\widehat{K}^{3 D}\right)}+p^{1 / 2}\|F u\|_{L^{2}\left(f_{e, 1} \cup f_{e, 2}\right)}\right)$.

To see this, we adapt the proof given in [31, Lemma B.5]. We split $\widehat{K}^{3 D}=K_{1} \cup K_{2}$ with $K_{i}=\left\{(x, y, z) \in \widehat{K}^{3 D} \mid d(x, y, z) \leq d_{i}(x, y, z)\right\}, i \in\{1,2\}$. We note that on $K_{1}$, we have $d(x, y, z)=d_{1}(x, y, z)=z$. Hence, by the arguments given in 31, Lemma B.5], we get

$$
p\|G u\|_{L^{2}\left(K_{1}\right)}+\|G u\|_{H^{1}\left(K_{1}\right)} \leq C\left(\|F u\|_{H^{1}\left(K_{1}\right)}+p^{1 / 2}\|F u\|_{L^{2}\left(f_{e, 1}\right)}\right) .
$$

Proceeding completely analogously for $K_{2}$ gives us (B.2). Since $\left.F u\right|_{\partial K^{3 D}}$ coincides with $E u$, we conclude that $G u$ satisfies

$$
p\|G u\|_{L^{2}\left(K_{1}\right)}+\|G u\|_{H^{1}\left(K_{1}\right)} \leq C p^{-1 / 2}\left(\|u\|_{H_{00}^{1 / 2}(e)}+p^{1 / 2}\|u\|_{L^{2}(e)}\right) .
$$

We recall that $\Pi_{p}^{H^{1}}: H_{0}^{1}\left(\widehat{K}^{3 D}\right) \rightarrow H_{0}^{1}\left(\widehat{K}^{3 D}\right) \cap \mathcal{P}_{p}$ denotes the $H^{1}$-projection and we define

$$
\pi_{e} u:=F u+\Pi_{p}^{H^{1}}(G u-F u) .
$$

If $u$ is a polynomial of degree $p$, then $\pi_{e} u$ is a polynomial of degree $p$. Additionally, $\pi_{e} u=F u$ on $\partial \widehat{K}^{3 D}$ so that the estimates for $\pi_{e}$ on the faces of $\widehat{K}^{3 D}$ are satisfied. To see the $H^{1}\left(\widehat{K}^{3 D}\right)$ - and $L^{2}\left(\widehat{K}^{3 D}\right)$-bounds we note that the stability of $\Pi_{p}^{H^{1}}$ together with (B.3) and the stability of $F$ gives us the $H^{1}$-bound. The $L^{2}$-bound follows as in the proof of Lemma B.1 and in 21] from Nitsche's trick: $\left\|\pi_{e} u\right\|_{L^{2}\left(\widehat{K}^{3 D}\right)} \leq$ $\left\|\pi_{e} u-G u\right\|_{L^{2}\left(\widehat{K}^{3 D}\right)}+\|G u\|_{L^{2}\left(\widehat{K}^{3 D}\right)} \leq C p^{-1}\|F u-G u\|_{H^{1}\left(\widehat{K}^{3 D}\right)}+\|G u\|_{L^{2}\left(\widehat{K}^{3 D}\right)}$.

B.2. Approximation operators. Lemma B.3 provides polynomial approximation results on triangles and tetrahedra. The lifting operators of the preceding subsection are employed in Theorem B.4 to modify the approximations of Lemma B.3 such that approximations are obtained that permit an element-by-element construction in the sense of Definition 5.3, that is, the approximation $\pi u$ of a function $u$ satisfies the following: For every vertex $V$, edge $e$, face $f$ of $\widehat{K}$, the restrictions $(\pi u)(V),\left.(\pi u)\right|_{e},\left.(\pi u)\right|_{f}$ are completely determined by $u(V),\left.u\right|_{\bar{e}},\left.u\right|_{\bar{f}}$, respectively.

Lemma B.3. Let $\widehat{K}$ be the reference triangle or the reference tetrahedron. Let $s>d / 2$. Then there exists for every $p$ a bounded linear operator $\pi_{p}: H^{s}(\widehat{K}) \rightarrow \mathcal{P}_{p}$ and for each $t \in[0, s]$ a constant $C>0$ (depending only on $s$ and $t$ ) such that

$$
\left\|u-\pi_{p} u\right\|_{H^{t}(\widehat{K})} \leq C p^{-(s-t)}|u|_{H^{s}(\widehat{K})}, \quad p \geq s-1 .
$$

Additionally, we have $\left\|u-\pi_{p} u\right\|_{L^{\infty}(\widehat{K})} \leq C p^{-(s-d / 2)}|u|_{H^{s}(\widehat{K})}$. Furthermore, for the case $d=2$ we have $\left\|u-\pi_{p} u\right\|_{H^{t}(e)} \leq C p^{-(s-1 / 2-t)}|u|_{H^{s}(\widehat{K})}$ for $0 \leq t \leq s-1 / 2$ for every edge. For the case $d=3$ we have $\left\|u-\pi_{p} u\right\|_{H^{t}(f)} \leq C p^{-(s-1 / 2-t)}|u|_{H^{s}(\widehat{K})}$ 
for $0 \leq t \leq s-1 / 2$ for every face $f$ and $\left\|u-\pi_{p} u\right\|_{H^{t}(e)} \leq C p^{-(s-1-t)}|u|_{H^{k}(\widehat{K})}$ for $0 \leq t \leq s-1$ for every edge.

Proof. The construction of $\pi_{p}$ with the property (B.4) is fairly classical (see, e.g., [5]). One possible construction is worked out in [31, Appendix A] first for integers $s, t$ and, then, interpolation arguments remove this restriction. Next, we consider the $L^{\infty}$-bound, for which we need the assumption $s>d / 2$ : We recall that for a Lipschitz domain $K \subset \mathbb{R}^{d}$ and $s>d / 2$ there exists $C>0$ such that

$$
\|u\|_{L^{\infty}(K)} \leq C\|u\|_{L^{2}(K)}^{1-d /(2 s)}\|u\|_{H^{s}(K)}^{d /(2 s)} \quad \forall u \in H^{s}(K) .
$$

From this, the desired $L^{\infty}$-bound follows easily. The inequality (B.5) can be seen as follows: First, using an extension operator for $K$ (e.g., the one given in 42, Chap. VI]) it suffices to show this estimate with $K$ replaced with the full space $\mathbb{R}^{d}$. Next, [45, Thm. 4.6.1] asserts the embedding $B_{2,1}^{d / 2}\left(\mathbb{R}^{d}\right) \subset C\left(\mathbb{R}^{d}\right)$. Finally, the Besov space $B_{2,1}^{d / 2}\left(\mathbb{R}^{d}\right)$ is recognized as an interpolation space between $L^{2}\left(\mathbb{R}^{d}\right)$ and $H^{s}\left(\mathbb{R}^{d}\right): B_{2,1}^{d / 2}\left(\mathbb{R}^{d}\right)=\left(L^{2}\left(\mathbb{R}^{d}\right), H^{s}\left(\mathbb{R}^{d}\right)\right)_{d /(2 s), 1}$. The interpolation inequality then produces the desired result. The remaining estimates on the edges and faces follow from appropriate trace inequalities. Specifically, let $\omega \subset \partial \widehat{K}$ be an edge (for $d=2$ ) or a face (for $d=3$ ). By [45, Thm. 2.9.3] the trace operator $\gamma$ is a continuous mapping in the following spaces:

$$
\gamma: B_{2,1}^{1 / 2}(\widehat{K}) \rightarrow L^{2}(\omega) \quad \text { and } \quad \gamma: H^{t}(\widehat{K}) \rightarrow H^{t-1 / 2}(\omega), \quad t>1 / 2 .
$$

Together with the observation $B_{2,1}^{1 / 2}(\widehat{K})=\left(L^{2}(\widehat{K}), H^{s}(\widehat{K})\right)_{1 /(2 s), 1}$ the desired estimates can be inferred. It remains to see the case of traces on an edge $e$ of the tetrahedron in the case $d=3$. In this case [45, Thm. 2.9.4] asserts the continuity of the trace operator in the following spaces:

$$
\gamma: B_{2,1}^{1}(\widehat{K}) \rightarrow L^{2}(e) \quad \text { and } \quad \gamma: H^{t}(\widehat{K}) \rightarrow H^{t-1}(e), \quad t>1 .
$$

Again, these continuity properties are sufficient to establish the desired error estimates.

We conclude this section with the construction of an approximation operator that permits an easy element-by-element construction.

Theorem B.4. Let $\widehat{K} \subset \mathbb{R}^{d}$ be the reference triangle or the reference tetrahedron. Let $s>d / 2$. Then there exists $C>0$ (depending only on $s$ and $d$ ) and for every $p$ a linear operator $\pi: H^{s}(\widehat{K}) \rightarrow \mathcal{P}_{p}$ that permits an element-by-element construction in the sense of Definition 5.3 such that

$$
p\|u-\pi u\|_{L^{2}(\widehat{K})}+\|u-\pi u\|_{H^{1}(\widehat{K})} \leq C p^{-(s-1)}|u|_{H^{s}(\widehat{K})} \quad \forall p \geq s-1 .
$$

Proof. We discuss only the case $d=3$; the case $d=2$ is treated very similarly. Also, we will construct $\pi u$ for a given $u$; inspection of the construction shows that $u \mapsto \pi u$ is in fact a linear operator. 
Let $\pi^{1} \in \mathcal{P}_{p}$ be given by Lemma B.3. Then, for $p \geq s-1$ there holds

$$
\begin{array}{ll}
\text { (B.7) }\left\|u-\pi^{1}\right\|_{H^{t}(\widehat{K})} \leq C p^{-(s-t)}|u|_{H^{s}(\widehat{K})}, \quad 0 \leq t \leq s, & \\
\text { (B.8) } \quad\left\|u-\pi^{1}\right\|_{H^{t}(f)} \leq C p^{-(s-t-1 / 2)}|u|_{H^{s}(\widehat{K})}, \quad \forall \text { faces } f, \quad 0 \leq t \leq s-1 / 2, \\
\text { (B.9) } \quad\left\|u-\pi^{1}\right\|_{H^{t}(e)} \leq C p^{-(s-t-1)}|u|_{H^{s}(\widehat{K})}, \quad \forall \text { edges } e, \quad 0 \leq t \leq s-1, \\
\text { (B.10) }\left\|u-\pi^{1}\right\|_{L^{\infty}(\widehat{K})} \leq C p^{-(s-3 / 2)}|u|_{H^{s}(\widehat{K})} .
\end{array}
$$

From (B.10) and the vertex-lifting properties given in Lemma B.2. we may adjust $\pi^{1}$ by vertex liftings to obtain a polynomial $\pi^{2}$ satisfying (B.7)-(B.9) and additionally the condition (5.3) of Definition 5.3. We next adjust the edge values. The polynomial $\pi^{2}$ coincides with $u$ in the vertices and satisfies (B.9). By fixing a $t \in(1 / 2, s-1)$, we get from an interpolation inequality:

$$
\begin{aligned}
& p^{1 / 2}\left\|u-\pi^{2}\right\|_{L^{2}(e)}+\left\|u-\pi^{2}\right\|_{H_{00}^{1 / 2}(e)} \\
& \quad \leq p^{1 / 2}\left\|u-\pi^{2}\right\|_{L^{2}(e)}+C\left\|u-\pi^{2}\right\|_{L^{2}(e)}^{1-1 /(2 t)}\left\|u-\pi^{2}\right\|_{H^{t}(e)}^{1 /(2 t)} \\
& \quad \leq C p^{-(s-3 / 2)}|u|_{H^{s}(\widehat{K})} .
\end{aligned}
$$

Hence, for an edge $e$, the minimizer $\pi^{e}$ of the functional (5.4) satisfies $p^{1 / 2} \| u-$ $\pi^{e}\left\|_{L^{2}(e)}+\right\| u-\pi^{e} \|_{H_{00}^{1 / 2}(e)} \leq C p^{-(s-3 / 2)}|u|_{H^{k}(\widehat{K})}$; the triangle inequality therefore gives that the correction $\pi^{e}-\pi^{2}$ needed to obtain condition (5.3) of Definition 5.3 likewise satisfies $p^{1 / 2}\left\|\pi^{e}-\pi^{2}\right\|_{L^{2}(e)}+\left\|\pi_{e}-\pi^{2}\right\|_{H_{00}^{1 / 2}(e)} \leq C p^{-(s-3 / 2)}|u|_{H^{s}(\widehat{K})}$. We conclude that the edge lifting of Lemma B.2 allows us to adjust $\pi^{2}$ to get a polynomial $\pi^{3} \in \mathcal{P}_{p}$ that satisfies the conditions (5.3) and (5.3) of Definition [5.3. Additionally, we have

$$
\begin{aligned}
& p\left\|u-\pi^{3}\right\|_{L^{2}(\widehat{K})}+\left\|u-\pi^{3}\right\|_{H^{1}(\widehat{K})} \leq C p^{-(s-1)}|u|_{H^{s}(\widehat{K})}, \\
& p\left\|u-\pi^{3}\right\|_{L^{2}(f)}+\left\|u-\pi^{3}\right\|_{H^{1}(f)} \leq C p^{-(s-3 / 2)}|u|_{H^{s}(\widehat{K})} \quad \text { for all faces } f .
\end{aligned}
$$

Since $\left.\pi^{3}\right|_{e}=\pi^{e}$ for the edges, the minimizer $\pi^{f}$ of the functional (5.5) for each face $f$ has to satisfy $p\left\|u-\pi^{f}\right\|_{L^{2}(f)}+\left\|u-\pi^{f}\right\|_{H^{1}(f)} \leq p\left\|u-\pi^{3}\right\|_{L^{2}(f)}+\left\|u-\pi^{3}\right\|_{H^{1}(f)} \leq$ $C p^{-(s-3 / 2)}|u|_{H^{s}(\widehat{K})}$. From the triangle inequality, we conclude that

$$
p\left\|\pi^{3}-\pi^{f}\right\|_{L^{2}(f)}+\left\|\pi^{3}-\pi^{f}\right\|_{H^{1}(f)} \leq C p^{-(s-3 / 2)}|u|_{H^{k}(\widehat{K})},
$$

together with $\pi^{3}-\pi^{f} \in H_{0}^{1}(f)$.

Hence, the face lifting of Lemma B.2 allows us to correct the face values to achieve also condition (5.3) of Definition 5.3. Lemma B.2 also implies that the correction is such that $(\underline{B .6})$ is true.

\section{Appendix C. Approximation by $h p$-Finite elements. CASE II: ANALYTIC REGULARITY}

In this section, we construct a polynomial approximation operator for analytic functions that permits element-by-element construction in the sense of Definition 5.3 and leads to exponential rates of convergence.

Lemma C.1. Let $d \in\{2,3\}$. Let $G_{1}, G \subset \mathbb{R}^{d}$ be bounded open sets. Assume that $g: \overline{G_{1}} \rightarrow \mathbb{R}^{d}$ satisfies $g\left(G_{1}\right) \subset G$. Assume additionally that $g$ is injective on $\overline{G_{1}}$, 
analytic on $G_{1}$ and satisfies

$$
\left\|\nabla^{p} g\right\|_{L^{\infty}\left(G_{1}\right)} \leq C_{g} \gamma_{g}^{p} p ! \quad \forall p \in \mathbb{N}_{0}, \quad\left|\operatorname{det}\left(g^{\prime}\right)\right| \geq c_{0}>0 \quad \text { on } G_{1} .
$$

Let $f$ be analytic on $G$ and satisfy, for some $C_{f}, \gamma_{f}, \kappa>0$,

$$
\left\|\nabla^{p} f\right\|_{L^{2}(G)} \leq C_{f} \gamma^{p} \max \{p, \kappa\}^{p} \quad \forall p \in \mathbb{N}_{0} .
$$

Then, the function $f \circ g$ is analytic on $G_{1}$ and there exist constants $C, \gamma_{1}>0$ that depend solely on $\gamma_{g}, C_{g}, c_{0}$, and $\gamma_{f}$ such that

$$
\left\|\nabla^{p}(f \circ g)\right\|_{L^{2}(G)} \leq C C_{f} \gamma_{1}^{p} \max \{p, \kappa\}^{p} \quad \forall p \in \mathbb{N}_{0} .
$$

Proof. This is essentially proved in [30. Lemma 4.3.1]; specifically, [30, Lemma 4.3.1] analyzes the case $d=2$ and states that $C, \gamma_{1}$ depends on the function $g$. Inspection of the proof shows that the case $d=3$ can be handled analogously and shows that the dependence on the function $g$ can be reduced to a dependence on $C_{g}, \gamma_{g}$, and $\gamma_{f}$.

Lemma C.2. Let $d \in\{1,2,3\}$, and let $\widehat{K} \subset \mathbb{R}^{d}$ be the reference simplex. Let $\bar{\gamma}$, $\widetilde{C}>0$ be given. Then there exist constants $C, \sigma>0$ that depend solely on $\bar{\gamma}$ and $\widetilde{C}$ such that the following is true: For any function $u$ that satisfies for some $C_{u}, h$, $R>0, \kappa \geq 1$ the conditions

$$
\left\|\nabla^{n} u\right\|_{L^{2}(\widehat{K})} \leq C_{u}(\bar{\gamma} h)^{n} \max \{n / R, \kappa\}^{n} \quad \forall n \in \mathbb{N}, \quad n \geq 2,
$$

and for any polynomial degree $p \in \mathbb{N}$ that satisfies

$$
h / R+\kappa h / p \leq \widetilde{C}
$$

there holds

$$
\inf _{\pi \in \mathcal{P}_{p}}\|u-\pi\|_{W^{2, \infty}(\widehat{K})} \leq C C_{u}\left[\left(\frac{h / R}{\sigma+h / R}\right)^{p+1}+\left(\frac{\kappa h}{\sigma p}\right)^{p+1}\right] .
$$

Proof. Let $\Pi_{1} u \in \mathcal{P}_{1}$ be the $L^{2}$-projection of $u$ onto the space $\mathcal{P}_{1}$. Set $\widetilde{u}:=u-\Pi_{1} u$. It suffices to approximate $\widetilde{u}$ from $\mathcal{P}_{p}$. By the lemma of Deny and Lions and (C.3) we have

$$
\begin{aligned}
\|\widetilde{u}\|_{L^{2}(\widehat{K})} & \leq C\left\|\nabla^{2} u\right\|_{L^{2}(\widehat{K})} \leq C C_{u}\left(1+(h / R)^{2}+(h \kappa)^{2}\right) \leq C C_{u} p^{2}, \\
\|\nabla \widetilde{u}\|_{L^{2}(\widehat{K})} & \leq C\left\|\nabla^{2} u\right\|_{L^{2}(\widehat{K})} \leq C C_{u}\left(1+(h / R)^{2}+(h \kappa)^{2}\right) \leq C C_{u} p h / R \max \{1, \kappa R\}, \\
\left\|\nabla^{n} \widetilde{u}\right\|_{L^{2}(\widehat{K})} & =\left\|\nabla^{n} u\right\|_{L^{2}(\widehat{K})} \leq C C_{u}(\bar{\gamma} h / R)^{n} \max \{n, R \kappa\}^{n} \quad \forall n \geq 2 .
\end{aligned}
$$

We conclude that (estimating generously $p \leq p^{2}$ for the case $n=1$ )

$$
\left\|\nabla^{n} \widetilde{u}\right\|_{L^{2}(\widehat{K})} \leq C C_{u} p^{2}(\bar{\gamma} h / R)^{n} \max \{n, \kappa R\}^{n} \quad \forall n \in \mathbb{N}_{0} .
$$

For the case $\kappa R \leq 1$, we estimate $\kappa R \leq 1$ and get directly from [30, Thm. 3.2.19]

$$
\inf _{\pi \in \mathcal{P}_{p}}\|\widetilde{u}-\pi\|_{W^{2, \infty}(\widehat{K})} \leq C C_{u}\left(\frac{h / R}{\sigma+h / R}\right)^{p+1} .
$$

It remains to consider the case $\kappa R>1$. To that end, we note that (C.5) and the Sobolev embedding theorem $H^{2}(\widehat{K}) \subset C(\overline{\widehat{K}})$ gives us for suitable $C>0$,

$$
\begin{aligned}
&\left\|\nabla^{n} \widetilde{u}\right\|_{L^{\infty}(\widehat{K})} \leq C_{u} p^{2} C {\left[(\bar{\gamma} h / R)^{n+2} \max \{n+2, \kappa R\}^{n+2}\right.} \\
&+\left.(\bar{\gamma} h / R)^{n} \max \{n+2, \kappa R\}^{n}\right] \\
& \leq C C_{u} p^{2}(\bar{\gamma} h / R)^{n} \max \{n+2, \kappa R\}^{n}\left(1+\max \{(n+2) h / R, h \kappa\}^{2}\right) \quad \forall n \in \mathbb{N}_{0} .
\end{aligned}
$$


Hence, we get for suitable constant $\gamma>0$ in view of (C.3),

$$
\left\|\nabla^{n} \widetilde{u}\right\|_{L^{\infty}(\widehat{K})} \leq C C_{u} p^{4}(\bar{\gamma} \gamma h / R)^{n} \max \{n+2, \kappa R\}^{n} \quad \forall n \in \mathbb{N}_{0} .
$$

Define

$$
\mu:=\gamma \bar{\gamma} \sqrt{d} \mathrm{e}
$$

and let $r_{0}=\operatorname{diam}(\widehat{K})$ and $b_{\widehat{K}}$ be the barycenter of $\widehat{K}$. The bounds (C.6), (5.2) and Stirling's formula in the form $n ! \geq(n / \mathrm{e})^{n}$ imply that the Taylor series of $\widetilde{u}$ about $x \in \widehat{K}$ converges on a (complex) ball $B_{1 /(\mu h / R)}(x) \subset \mathbb{C}^{d}$ of radius $1 /(\mu h / R)$ and center $x \in \widehat{K}$. For the polynomial approximation of $\widetilde{u}$, we distinguish the cases $\mu h / R \leq 1 /\left(2 r_{0}\right)$ and $\mu h / R>1 /\left(2 r_{0}\right)$.

The case $\mu h / R \leq 1 /\left(2 r_{0}\right)$ : In this case the Taylor series of $\widetilde{u}$ about $b_{\widehat{K}}$ converges on an open ball that contains the closure of $\widehat{K}$. We may therefore approximate $\widetilde{u}$ by its truncated Taylor series $T_{p} u$. The error is then given by

$$
\widetilde{u}(x)-T_{p} u(x)=\sum_{\alpha \in \mathbb{N}_{0}^{d}:|\alpha| \geq p+1} \frac{1}{\alpha !} D^{\alpha} \widetilde{u}\left(b_{\widehat{K}}\right)\left(x-b_{\widehat{K}}\right)^{\alpha}, \quad x \in B_{1 /(\mu h / R)}\left(b_{\widehat{K}}\right) \subset \mathbb{C}^{d} .
$$

Hence (5.2) and (C.6) imply

$$
\begin{aligned}
\left\|\widetilde{u}-T_{p} u\right\|_{L^{\infty}\left(B_{r_{0}}\left(b_{\widehat{K}}\right)\right)} & \leq \sum_{|\alpha| \geq p+1} \frac{1}{\alpha !}\left|D^{\alpha} \widetilde{u}\left(b_{\widehat{K}}\right)\right| r_{0}^{|\alpha|} \leq \sum_{n=p+1}^{\infty} r_{0}^{n} d^{n / 2} \frac{1}{n !}\left\|\nabla^{n} \widetilde{u}\right\|_{L^{\infty}(\widehat{K})} \\
& \leq C C_{u} p^{4} \sum_{n=p+1}^{\infty} \frac{1}{n !} \max \{n+2, \kappa R\}^{n} d^{n / 2}(\gamma \bar{\gamma} h / R)^{n} r_{0}^{n}=: S .
\end{aligned}
$$

The last sum $S$ is split further using Stirling's formula $n ! \geq(n / \mathrm{e})^{n}$ and $(1+2 / n)^{n} \leq$ $\mathrm{e}^{2}$ :

$$
\begin{aligned}
S=C C_{u} p^{4}\left(\sum_{p+1 \leq n \leq \kappa R-2} \frac{1}{n !}\left(\sqrt{d} r_{0} \gamma \bar{\gamma} \kappa h\right)^{n}\right. & \left.+\sum_{n \geq \max \{p+1, \kappa R-2\}}\left(\gamma \bar{\gamma} r_{0} \sqrt{d} h / R\right)^{n} \frac{(n+2)^{n}}{n !}\right) \\
& \leq C C_{u} p^{4} \mathrm{e}^{2}\left(\sum_{n \geq p+1} \frac{1}{n !}\left(\sqrt{d} r_{0} \gamma \bar{\gamma} \kappa h\right)^{n}+\sum_{n \geq p+1}(\underbrace{\mathrm{e} \gamma \bar{\gamma} \sqrt{d}}_{=\mu} r_{0} h / R)^{n}\right)=: S_{1}+S_{2} .
\end{aligned}
$$

We estimate these two sums separately. For $S_{2}$, we use the assumption $\mu r_{0} h / R \leq$ $1 / 2$, which allows us to estimate

$$
\begin{aligned}
S_{2} \leq C C_{u} p^{4} \mathrm{e}^{2}\left(\mu r_{0} h / R\right)^{p+1} & =C C_{u} p^{4} \mathrm{e}^{2}\left(\frac{h / R}{\frac{1}{2 \mu r_{0}}+\frac{1}{2 \mu r_{0}}}\right)^{p+1} \\
& \leq C C_{u} p^{4} \mathrm{e}^{2}\left(\frac{h / R}{\frac{1}{2 \mu r_{0}}+h / R}\right)^{p+1} .
\end{aligned}
$$


For $S_{1}$, we recall that Taylor's formula gives, for $x>0$,

$$
\sum_{n \geq p+1} \frac{1}{n !} x^{n}=\mathrm{e}^{x}-\sum_{n=0}^{p} \frac{1}{n !} x^{n}=\frac{1}{p !} \int_{0}^{x}(x-t)^{p} \mathrm{e}^{t} d t \leq \frac{x^{p+1}}{p !} \mathrm{e}^{x} .
$$

Hence, we can estimate $S_{1}$ by (recall that $\bar{\gamma} \gamma \sqrt{d}=\mu / \mathrm{e}$ ),

$$
S_{2} \leq C C_{u} p^{4} \frac{\left((\mu / \mathrm{e}) r_{0} \kappa h\right)^{p+1}}{p !} \mathrm{e}^{(\mu / \mathrm{e}) r_{0} \kappa h} \leq C C_{u} p^{5}\left(\frac{\mathrm{e}^{\theta} \mu r_{0} \kappa h}{p+1}\right)^{p+1}
$$

where, in the second inequality, we have used the assumption $h \kappa / p \leq \widetilde{C}$ and Stirling's formula $n ! \geq(n / \mathrm{e})^{n}$ and have abbreviated $\theta:=\widetilde{C} \mu / \mathrm{e} r_{0}$. Combining the estimates for $S_{1}$ and $S_{2}$ we arrive at the following estimate for suitable $\sigma>0$ (depending only on $\mu, r_{0}$, and $\widetilde{C}$ ):

$$
\left\|\widetilde{u}-T_{p} u\right\|_{L^{\infty}\left(B_{r_{0}}\left(b_{\widehat{K}}\right)\right)} \leq S \leq C C_{u}\left(\left(\frac{\kappa h}{\sigma p}\right)^{p+1}+\left(\frac{h / R}{\sigma+h / R}\right)^{p+1}\right) .
$$

Since $\operatorname{dist}\left(\widehat{K}, \partial B_{r_{0}}\left(b_{\widetilde{K}}\right)\right)>0$, the Cauchy integral formula for derivatives then implies

$$
\left\|\widetilde{u}-T_{p} u\right\|_{W^{2, \infty}(\widehat{K})} \leq C C_{u}\left(\left(\frac{\kappa h}{\sigma p}\right)^{p+1}+\left(\frac{h / R}{\sigma+h / R}\right)^{p+1}\right) .
$$

The case $\mu h / R>1 /\left(2 r_{0}\right)$ : We recall that for every $x \in \widehat{K}$ the Taylor series of $\widetilde{u}$ about $x$ converges on the (complex) ball $B_{1 /(\mu h / R)}(x) \subset \mathbb{C}^{d}$. From (C.3) we get a lower bound for $1 /(\mu h / R)$, namely, $1 /(\mu h / R) \geq 1 /(\mu \widetilde{C})=: 2 r_{1}$. We conclude that $\widetilde{u}$ is analytic on $\widehat{\mathcal{U}}_{2 r_{1}}:=\bigcup_{x \in \widehat{K}} B_{2 r_{1}}(x) \subset \mathbb{C}^{d}$. The estimate (C.6) and a calculation analogous to the above reveals that on $\widehat{\mathcal{U}}_{r_{1}}:=\bigcup_{x \in \widehat{K}} B_{r_{1}}(x)$ we have

$$
\|\widetilde{u}\|_{L^{\infty}\left(\hat{\mathcal{U}}_{r_{1}}\right)} \leq C C_{u} p^{4} \mathrm{e}^{\vartheta \kappa h}
$$

for a constant $\vartheta>0$ independent of $p, \kappa, h$. Approximation results for analytic functions on triangles/tetrahedra (see [30, Prop. 3.2.16] for the case $d=2$ and [16, Thm. 1] for the case $d=3$ ) imply the existence of $C, b>0$ that depend solely on $r_{1}$ such that

$$
\inf _{\pi \in \mathcal{P}_{p+1}}\|u-\pi\|_{W^{2, \infty}(\widehat{K})} \leq C C_{u} p^{4} \mathrm{e}^{\vartheta \kappa h} \mathrm{e}^{-b p} \quad \forall p \in \mathbb{N}_{0} .
$$

We finally distinguish two further cases: If $\vartheta \kappa h<p b / 2$, then we can estimate

$$
p^{4} \mathrm{e}^{\vartheta \kappa h p} \mathrm{e}^{-b p} \leq p^{4} \mathrm{e}^{-b / 2 p} \leq C\left(\frac{1 /\left(2 \mu r_{0}\right)}{\sigma+1 /\left(2 \mu r_{0}\right)}\right)^{p+1},
$$

for suitable constants $C, \sigma>0$ depending only on $b, \mu$, and $r_{0}$. Since $h / R \geq$ $1 /\left(2 \mu r_{0}\right)$ and the function $x \mapsto x /(\sigma+x)$ is monotone increasing, we have reached the desired bound. If, on the other hand, $\vartheta \kappa h \geq p b / 2$, then

$$
p^{4} \mathrm{e}^{\vartheta \kappa h} \mathrm{e}^{-b p} \leq C \mathrm{e}^{\vartheta \kappa h} \leq C \mathrm{e}^{\vartheta \widetilde{C} p}=C\left(\mathrm{e}^{\vartheta \widetilde{C}}\right)^{p} \leq C\left(\frac{\kappa h}{p} \frac{2}{b} \mathrm{e}^{\vartheta \widetilde{C}}\right)^{p} ;
$$

we recognize this bound to have the desired form. 
Lemma C.3. Assume the hypotheses of Lemma C.2. Then one can find a polynomial $\pi \in \mathcal{P}_{p}$ that satisfies

$$
\|u-\pi\|_{W^{1, \infty}(\widehat{K})} \leq C C_{u}\left[\left(\frac{h / R}{\sigma+h / R}\right)^{p+1}+\left(\frac{\kappa h}{\sigma p}\right)^{p+1}\right]
$$

and additionally admits an element-by-element construction as defined in Definition 5.3 .

Proof. The construction follows standard lines. We will only outline the arguments for the case $d=3$. In order to keep the notation compact, we introduce the expression

$$
E(C, \sigma):=C C_{u}\left[\left(\frac{h / R}{\sigma+h / R}\right)^{p+1}+\left(\frac{\kappa h}{\sigma p}\right)^{p+1}\right] .
$$

In what follows, the constants $C_{i}, \sigma_{i}>0(i=1,2, \ldots)$ will be independent of $C_{u}, h, R, p$, and $\kappa$. Let $\pi \in \mathcal{P}_{p}$ be the polynomial given by Lemma C.2 It satisfies $\|u-\pi\|_{W^{2, \infty}(\widehat{K})} \leq E(C, \sigma)$. Therefore, we may correct $\pi$ by a linear polynomial without sacrificing the approximation rate to ensure $u(V)-\pi(V)$ for all vertices $V \in \mathcal{V}$. This corrected polynomial, denoted $\pi^{2}$, vanishes in the vertices and still satisfies $\left\|u-\pi^{2}\right\|_{W^{2, \infty}(\widehat{K})} \leq E\left(C_{2}, \sigma_{2}\right)$. Next, we correct the edges. We illustrate the procedure only for one edge. Without loss of generality, we assume that $\widehat{K}=\{(x, y, z) \mid 0<x, y, z<1, x+y<1-z\}$ and that the edge $e$ considered is $e=\{(0,0, z) \mid z \in(0,1)\}$. Let the univariate polynomial $\pi^{e} \in \mathcal{P}_{p}$ be the minimizer of the functional (5.4). From $\left\|u-\pi^{2}\right\|_{W^{2, \infty}(e)} \leq\left\|u-\pi^{2}\right\|_{W^{2, \infty}(\widehat{K})} \leq E\left(C_{2}, \sigma_{2}\right)$ we can conclude that $p^{1 / 2}\left\|u-\pi^{e}\right\|_{L^{2}(e)}+\left\|u-\pi^{e}\right\|_{H_{00}^{1 / 2}(e)} \leq C p^{1 / 2} E\left(C_{2}, \sigma_{2}\right)$. Hence, for the required correction $\pi^{c}:=\left.\pi^{2}\right|_{e}-\pi^{e}$, which vanishes in the two endpoints of $e$, we get from a triangle inequality and standard polynomial inverse estimates $\left\|\frac{1}{1-z} \pi^{c}\right\|_{L^{\infty}(e)}+\left\|\pi^{c}\right\|_{L^{\infty}(e)} \leq E\left(C_{3}, \sigma_{3}\right)$. We may lift this univariate function to $\widehat{K}$ by

$$
\tilde{\pi}^{e}(x, y, z):=\frac{1-x-y-z}{1-z} \pi^{c}(z) .
$$

This is a polynomial of degree $\leq p$ that vanishes on all edges but the edge $e$; clearly, $\left\|\tilde{\pi}^{e}\right\|_{L^{\infty}(\widehat{K})} \leq E\left(C_{3}, \sigma_{3}\right)$. The polynomial inverse estimate $\left\|\tilde{\pi}^{e}\right\|_{W^{1, \infty}(\widehat{K})} \leq$ $C p^{2}\left\|\widetilde{\pi}^{e}\right\|_{L^{\infty}(\widehat{K})}$ shows that $\left\|\widetilde{\pi}^{e}\right\|_{W^{2, \infty}(\widehat{K})} \leq E\left(C_{4}, \sigma_{4}\right)$. Proceeding in this fashion for all edges, we arrive at a polynomial $\pi^{3}$ with the desired behavior on all edges of $\widehat{K}$ and satisfies $\left\|u-\pi^{3}\right\|_{W^{2, \infty}(\widehat{K})} \leq E\left(C_{5}, \sigma_{5}\right)$.

It remains to construct a correction for the faces. To that end, the key issue is again that of a lifting from a face $f$. Without loss of generality, this face is $f:=\{(x, y, 0) \mid 0<x, y, x+y<1\}$. For a polynomial $\pi^{c}$ defined on $f$ that additionally vanishes on $\partial f$, we define the lifting $\widetilde{\pi}^{f}$ by

$$
\tilde{\pi}^{f}(x, y, z)=\frac{x y(1-x-y-z)}{x y(1-x-y)} \pi^{c}(x, y) .
$$

This is a polynomial that vanishes on all faces of $\widehat{K}$ except on $f$. Additionally, it is a lifting, i.e., $\left.\widetilde{\pi}^{f}\right|_{f}=\pi^{c}$. As in the case of the lifting from the edge we see that if $\pi^{c}$ is exponentially small on $f$, then the lifting is likewise exponentially small. To see that the required correction $\pi^{c}$ is exponentially small, let $\pi^{f}$ be the minimizer of the functional (5.5). Since $\pi^{3}$ has the desired behavior on the edges of $f$, we have 
$\left.\pi^{3}\right|_{\partial f}=\left.\pi^{f}\right|_{\partial f}$ and therefore $\left\|u-\pi^{3}\right\|_{W^{2, \infty}(\widehat{K})} \leq E\left(C_{5}, \sigma_{5}\right)$ allows us to conclude $\left\|\pi^{3}-\pi^{f}\right\|_{H^{1}(f)} \leq C E\left(C_{5}, \sigma_{5}\right)$. Polynomial inverse estimates then imply for the lifting $\tilde{\pi}^{f}$ that $\left\|\tilde{\pi}^{f}\right\|_{W^{1, \infty}(\widehat{K})} \leq E\left(C_{6}, \sigma_{6}\right)$.

\section{ACKNOWLEDGMENT}

We would like to thank Professor R. Hiptmair for discussions concerning the choice of the model problem.

\section{REFERENCES}

[1] M. Abramowitz and I. A. Stegun. Handbook of Mathematical Functions. Applied Mathematics Series 55. National Bureau of Standards, U.S. Department of Commerce, 1972.

[2] M. Ainsworth. Discrete dispersion relation for $h p$-version finite element approximation at high wave number. SIAM J. Numer. Anal., 42(2):553-575, 2004. MR2084226 (2005i:65174)

[3] I. Babuška, A. Craig, J. Mandel, and J. Pitkäranta. Efficient preconditioning for the $p$ version finite element method in two dimensions. SIAM J. Numer. Anal., 28(3):624-661, 1991. MR.1098410 (92a:65282)

[4] I. Babuška and S. Sauter. Is the pollution effect of the FEM avoidable for the Helmholtz equation considering high wave numbers. SIAM, J. Numer. Anal., 34(6):2392-2423, 1997. MR:1480387 (99b:65135)

[5] I. Babuška and M. Suri. The optimal convergence rate of the $p$-version of the finite element method. SIAM J. Numer. Anal., 24:750-776, 1987. MR899702 (88k:65102)

[6] I. M. Babuška, F. Ihlenburg, E. T. Paik, and S. A. Sauter. A generalized finite element method for solving the Helmholtz equation in two dimensions with minimal pollution. Comp. Meth. Appl. Mech. Eng., 128:325-359, 1995. MR.1368049 (96j:65115)

[7] L. Banjai and S. Sauter. A Refined Galerkin Error and Stability Analysis for Highly Indefinite Variational Problems. SIAM J. Numer. Anal., 45(1):37-53, 2007. MR2285843 (2008c:65308)

[8] S. Brenner and L. Scott. The Mathematical Theory of Finite Element Methods. SpringerVerlag, New York, 1994. MR1278258 (95f:65001)

[9] A. Buffa and P. Monk. Error estimates for the ultra weak variational formulation of the Helmholtz equation. Math. Mod. Numer. Anal., pages 925-940, 2008. MR2473314 (2009j:65295)

[10] O. Cessenat and B. Després. Application of an ultra weak variational formulation of elliptic PDEs to the two-dimensional Helmholtz equation. SIAM J. Numer. Anal., 35:255-299, 1998. MR:1618464 (99b:65139)

[11] O. Cessenat and B. Després. Using plane waves as base functions for solving time harmonic equations with the ultra weak variational formulation. J. Computational Acoustics, 11:227238, 2003. MR2013687(2004j:65182)

[12] S. Chandler-Wilde and P. Monk. Wave-number-explicit bounds in time-harmonic scattering. SIAM J. Numer. Anal., pages 1428-1455, 2008. MR2377284 (2008k:35071)

[13] P. Ciarlet. The finite element method for elliptic problems. North-Holland, 1987. MR 0520174 $(58: 25001)$

[14] L. Demkowicz. Polynomial exact sequences and projection-based interpolation with applications to Maxwell's equations. In D. Boffi, F. Brezzi, L. Demkowicz, L. Durán, R. Falk, and M. Fortin, editors, Mixed Finite Elements, Compatibility Conditions, and Applications, volume 1939 of Lectures Notes in Mathematics. Springer-Verlag, 2008.

[15] A. Deraemaeker, I. Babuška, and P. Bouillard. Dispersion and pollution of the FEM solution for the Helmholtz equation in one, two and three dimesnions. Int. J. Numer. Meth. Eng., 46(4), 1999.

[16] T. Eibner and J. Melenk. An adaptive strategy for $h p$-FEM based on testing for analyticity. Computational Mechanics, 39:575-595, 2007. MR2288643 (2008d:65132)

[17] C. Farhat, I. Harari, and U. Hetmaniuk. A discontinuous Galerkin method with Lagrange multipliers for the solution of Helmholtz problems in the mid-frequency regime. Comp. Meth. Appl. Mech. Eng., 192:1389-1419, 2003. MR1963058 
[18] C. Farhat, R. Tezaur, and P. Weidemann-Goiran. Higher-order extensions of discontinuous Galerkin method for mid-frequency Helmholtz problems. Int. J. Numer. Meth. Eng., 61, 2004. MR2099956 (2005i:65185)

[19] C. Gittelson, R. Hiptmair, and I. Perugia. Plane wave discontinuous Galerkin methods. Technical Report NI07088-HOP, Isaac Newton Institute Cambridge, Cambridge, UK, 2007.

[20] I. S. Gradshteyn and I. Ryzhik. Table of Integrals, Series, and Products. Academic Press, New York, London, 1965. MR0197789 (33:5952)

[21] B. Guo and J. Zhang. Stable and compatible polynomial extensions in three dimensions and applications to the p and h-p finite element method. SIAM J. Numer. Anal., 47(2):1195-1225, 2009. MR 2485450

[22] I. Harari. Reducing spurious dispersion, anisotropy and reflection in finite element analysis of time-harmonic acoustics. Comput. Methods Appl. Mech. Engrg., 140(1-2):39-58, 1997. MR 1423456 (97g:76054)

[23] I. Harari. Finite element dispersion of cylindrical and spherical acoustic waves. Comput. Methods Appl. Mech. Engrg., 190(20-21):2533-2542, 2001.

[24] I. Harari and D. Avraham. High-order finite element methods for acoustic problems. J. Comput. Acoust., 5(1):33-51, 1997.

[25] I. Harari and T. Hughes. Finite element methods for the Helmholtz equation in an exterior domain: Model problems. Computer Methods in Applied Mechanics and Engineering, 87:5996, North Holland, 1991. MR.1103417 (92d:65192)

[26] T. Huttunen and P. Monk. The use of plane waves to approximate wave propagation in anisotropic media. J. Computational Mathematics, 25:350-367, 2007. MR 2320239 (2009b:78012)

[27] F. Ihlenburg. Finite Element Analysis of Acousting Scattering. Springer, New York, 1998. MR.1639879 (99g:65114)

[28] F. Ihlenburg and I. Babuška. Finite element solution to the Helmholtz equation with high wave number. Part II: The h-p version of the FEM. Siam J. Num. Anal., 34(1):315-358, 1997. MR1445739 (98f:65109)

[29] W. McLean. Strongly Elliptic Systems and Boundary Integral Equations. Cambridge, Univ. Press, 2000. MR:1742312 (2001a:35051)

[30] J. Melenk. hp finite element methods for singular perturbations, volume 1796 of Lecture Notes in Mathematics. Springer-Verlag, 2002. MR1939620 (2003i:65108)

[31] J. Melenk. $h p$-interpolation of nonsmooth functions and an application to $h p$ a posteriori error estimation. SIAM J. Numer. Anal., 43:127-155, 2005. MR2177138 (2006g:65178)

[32] J. Melenk and S. Sauter. Wave-number explicit convergence analysis for finite element discretizations of the Helmholtz equation. Technical Report 09/2009, Inst. für Mathematik, Univ. Zürich, 2009.

[33] J. M. Melenk. On Generalized Finite Element Methods. Ph.D. thesis, University of Maryland at College Park, 1995.

[34] R. Muñoz-Sola. Polynomial liftings on a tetrahedron and applications to the $h p$-version of the finite element method in three dimensions. SIAM J. Numer. Anal., 34(1):282-314, 1997. MR 1445738 (98k:65069)

[35] J. C. Nédélec. Acoustic and Electromagnetic Equations. Springer, New York, 2001.

[36] A. Oberai and P. Pinsky. A numerical comparison of finite element methods for the Helmholtz equation. J. Comput. Acoust., 8(1):211-221, 2000. MR1768338

[37] F. Olver. Asymptotics and Special Functions. A K Peters, Natick, 1997. MR1429619 (97i:41001)

[38] E. Perrey-Debain, O. Laghrouche, and P. Bettess. Plane-wave basis finite elements and boundary elements for three-dimensional wave scattering. Phil. Trans. R. Soc. London A, 362:561577, 2004. MR2075907 (2005b:78017)

[39] S. Sauter. A refined finite element convergence theory for highly indefinite Helmholtz problems. Computing, 78(2):101-115, 2006. MR2255368 (2007h:65130)

[40] A. Schatz. An obeservation concerning Ritz-Galerkin methods with indefinite bilinear forms. Math. Comp., 28:959-962, 1974. MR0373326 (51:9526)

[41] C. Schwab. p- and hp-Finite Element Methods. Oxford University Press, 1998. MR.1695813 (2000d:65003)

[42] E. Stein. Singular integrals and differentiability properties of functions. Princeton University Press, 1970. MR0290095 (44:7280) 
[43] M. Stojek. Least-squares Trefftz-type elements for the Helmholtz equation. Int. J. Numer. Meth. Engr., 41:831-849, 1998. MR1607804

[44] R. Tezaur and C. Farhat. Three-dimensional discontinuous Galerkin elements with plane waves and Lagrange multipliers for the solution of mid-frequency Helmholtz problems. Int. J. Numer. Meth. Engr., 66:796-815, 2006. MR2219901(2007a:65215)

[45] H. Triebel. Interpolation Theory, Function Spaces, Differential Operators. Johann Ambrosius Barth, 2 edition, 1995. MR.1328645 (96f:46001)

[46] G. N. Watson. A Treatise on the Theory of Bessel Functions. Cambridge University Press, 1922. MR1349110(96i:33010)

Institut für Analysis und Scientific Computing, Technische Universität Wien, Wiedner Hauptstrasse 8-10, A-1040 Wien, Austria

E-mail address: melenk@tuwien.ac.at

Institut Für Mathematik, Universität Zürich, Winterthurerstr 190, CH-8057 Zürich, SWITZERLAND

E-mail address: stas@math.uzh.ch 